\title{
1 Amoeba predation of Cryptococcus neoformans results in pleiotropic 2 changes to traits associated with virulence
}

3 Man Shun $\mathrm{Fu}^{1}$, Livia C. Liporagi-Lopes ${ }^{1,2}$, Samuel R. dos Santos Júnior ${ }^{1,3}$, Jennifer L. Tenor ${ }^{4}$,

4 John R. Perfect ${ }^{4}$, Christina A. Cuomo ${ }^{5}$, Arturo Casadevall ${ }^{1}$

$5 \quad{ }^{1}$ Department of Molecular Microbiology and Immunology, Johns Hopkins Bloomberg School of

6 Public Health, Maryland, USA.

$7 \quad 2$ Departamento de Análises Clínicas e Toxicológicas, Faculdade de Farmácia, Universidade

$8 \quad$ Federal do Rio de Janeiro, Rio de Janeiro, Brazil.

$9{ }^{3}$ Departamento de Microbiologia, Instituto de Ciências Biomédicas, Universidade de São Paulo, 10 São Paulo, Brazil.

$11{ }^{4}$ Division of Infectious Diseases, Department of Medicine and Department of Molecular

12 Genetics and Microbiology, Duke University, North Carolina, USA.

$13{ }^{5}$ Infectious Disease and Microbiome Program, Broad Institute, Cambridge, Massachusetts, USA.

14 *Address correspondence to: Arturo Casadevall, M.D., Ph.D.

Johns Hopkins Bloomberg School of Public Health

$615 \mathrm{~N}$ Wolfe St

Baltimore, MD 21205

Telephone: (410) 955-3459

Fax: (410) 502-5884

Email: acasade1@jhu.edu

Running title: Amoeba predation of C. neoformans results in pleiotropic traits 
Abstract

Phagocytic amoeboid predators such as amoeba have been proposed to select for survival traits in soil microbes such as Cryptococcus neoformans that can also function in animal virulence by defeating phagocytic immune cells, such as macrophages. Several prior studies have shown that incubation of various fungal species with amoeba can enhance their virulence. However, the mechanisms by which fungi adapt to amoeba and thus change their virulence are unknown. In this study we exposed three strains of $C$. neoformans (1 clinical and 2 environmental) to predation by Acanthamoeba castellanii for prolonged periods of time and then analyzed surviving colonies phenotypically and genetically. Surviving colonies were comprised of cells that expressed either pseudohyphal or yeast phenotypes, which demonstrated variable expression of such traits associated with virulence such as capsule size, urease production and melanization. Phenotypic changes were associated with aneuploidy and DNA sequence mutations in some amoeba-passaged isolates, but not in others. Mutations in the gene encoding for the oligopeptide transporter (CNAG_03013; OPT1) were observed among amoeba-passaged isolates from each of the three strains. In addition, isolates derived from environmental strains gained the capacity for enhanced macrophage toxicity after amoeba selection and carried mutations on the CNAG_00570 gene, which encodes Pkr1 (AMP-dependent protein kinase regulator) but were less virulence in mice because they elicited more effective fungal-clearing immune responses. Our results indicate that $C$. neoformans survival under constant amoeba predation involves the generation of strains expressing pleiotropic phenotypic and genetic changes, which confer increase resistance against protozoal predation. Given the myriad of potential predators in soils the diversity observed among amoeba-selected strains suggests 

available under aCC-BY 4.0 International license.

47 a bet-hedging strategy whereby variant diversity increases the likelihood that some will

48 survive predation.

49 


\section{Author summary}

51 Cryptococcus neoformans is a ubiquitous environmental fungus that is also a leading cause of

52 fatal fungal infection in humans, especially among immunocompromised patients.

53 Cryptococcosis is a worldwide concern due to its high mortality rate. A major question in the

54 field is how an environmental yeast such as $C$. neoformans becomes a human pathogen when it

55 has no need for animal host in its life cycle. Previous studies showed evidence that $C$.

56 neoformans increases its pathogenicity after interacting with its environmental predator

57 amoebae. Amoebae behave like macrophages, an important immune cell in human body, so it is

58 considered as a training ground for pathogens to resist macrophages. However, how $C$.

59 neoformans changes its virulence through interacting with amoebae is unknown. Here, we

60 exposed $C$. neoformans to amoebae for a long period of time. We found that $C$. neoformans cells

61 recovered from amoebae manifested numerous changes to phenotypes related to its virulence and

62 one of the amoeba-passaged $C$. neoformans cells had enhanced ability to kill macrophages. We

63 further analyzed their genome sequences and found various mutations in different cells of

64 amoeba-passaged $C$. neoformans, showing that DNA mutations may be the major cause of the

65 phenotypic changes after interacting with amoebae. Our study indicates that fungal survival in

66 the face of amoeba predation is associated with the emergence of pleiotropic phenotypic and

67 genomic changes that increase the chance of fungal survival. 


\section{Introduction}

70

71

72

73

74

75

76

77

78

79

80

81

82

83

84

85

86

87

88

89

90

C. neoformans is a major life-threatening fungal pathogen that predominantly infects severely immunocompromised patients and causes over 180,000 deaths per year worldwide [1]. C. neoformans expresses virulence factors that promote its pathogenicity in humans, including formation and enlargement of a polysaccharide capsule that interferes with the host immune system in varied ways, melanin production that protects against oxidative stress [2-5], and extracellular secretion of various enzymes including phospholipase and urease [6,7]. $C$. neoformans is found primarily and ubiquitously in environments such as soils contaminated with bird excreta or from trees [8-11]. It is a saprophyte and does not require an animal host for survival and reproduction. Besides, there is rare evidence of human-to-human transmission and thus it is unlikely that its virulence traits were selected for causing disease in humans or animals. That raises the fundamental question of how $C$. neoformans acquired those traits, which are essential for pathogenesis of cryptococcosis in human.

A hypothesis to solve this enigma is that of coincident selection, resulting from selective pressures in both natural environmental and animal niches such as predatory amoeba and nematodes [12]. According to this view, microbial traits selected for environmental survival also confer the capacity for virulence by promoting survival in animal hosts [12]. For example, the capsule can protect the fungi from desiccation and against predatory amoeba $[13,14]$ while melanin may reduce damage of fungi from the exposure to UV radiation, osmotic stresses or extreme temperatures [15-18]. Urease provides a nutritional role involved in nitrogen acquisition in the environment [19]. Moreover, it is striking that $C$. neoformans isolates from the soil are virulent for animal hosts [20]. Understanding the evolutionary adaption of C. neoformans in 
91 nature will help us to understand further the origin of virulence and pathogenesis of

92

93

94

95

96

97 cryptococcosis.

Amoebae are one of the major sources of selective pressure in nature for broad range of soil microorganisms that have pathogenic potential for humans, including bacteria such as Legionella pneumophila, Mycobacterium spp and fungi such as Cryptococcus neoformans, Aspergillus fumigatus and Paracoccidioides spp [14,21-24]. Similar to human macrophages, amoebae ingest microorganisms, undergo a respiratory burst, phagosome maturation and acidification, expresses cell surface receptors and expel undigested materials [25-31]. However, many bacteria and fungi have strategies to survive in amoebae, that function in parallel for survival in mammalian phagocytic cells. For example, L. pneumophila utilizes similar cellular and molecular mechanisms of invasion, survival and replication inside both amoeba and macrophages [32-37]. Amoeba-grown L. pneumophila are more invasive for epithelial cells and macrophages [21]. After passage in amoeba, Mycobacterium avium enhances both entry and intracellular replication in epithelial cells and is more virulent in the macrophage and mouse models of infection [22]. Among fungal pathogens, concordance of virulence factor function for amoeba and animals was also demonstrated for A. fumigatus [23]. For example, the mycotoxin fumagillin can inhibit the growth of Entamoeba histolytica while it can also cause mammalian epithelial cell damage [38]. Many studies have been done to explore amoeba-C. neoformans interaction, and shown evidence that amoebae influence the virulence of $C$. neoformans for mammalian infection $[39,40]$.

Acanthamoeba castellanii was originally isolated from cultures of a Cryptococcus spp., and like other amoebae species preys on Cryptococcus spp [41,42]. There is evidence that amoebae are natural predators of $C$. neoformans in the natural environment [43]. On the other hand, $C$. 
114

115

116

117

118

119

120

121

122

123

124

125

126

127

128

129

130

131

132

133

134

135

neoformans is able to resist the destruction by amoeba, especially in nutrient poor conditions [44] without metal cations [45]. Several studies have shown that the virulence factors and the cellular process that fungi use for defending against amoeba predation are remarkably similar to those employed for mammalian virulence. For example, the capsule formation and melanin production are important for C. neoformans to resist predation by A. castellanii and play important roles for pathogenicity in mammalian infection [14,39]. Interestingly, the phospholipids that are secreted by both macrophages and amoebae trigger capsule enlargement [40]. The non-lytic exocytosis process which is found in macrophage containing C. neoformans can be also observed in A. castellanii and D. discoideum through the similar action of actin polymerization $[46,47]$. Transcriptional studies showed a conserved metabolic response of $C$. neoformans to the microenvironments of both macrophage and amoebae [48]. All those common strategies found to adapt to both amoebae and macrophages support the hypothesis that cryptococcal pathogenesis is derived from the interaction with amoebae in natural environment. More direct evidence comes from the experiment on the passage of an attenuated cryptococcal strain to $D$. discoideum cultures that shows enhancement of fungal virulence in a murine infection model [39]. Passaged C. neoformans also exhibits capsule enlargement and rapid melanization, suggesting that those are mechanisms to enhance the survival of fungus in mice. However, the underlying mechanism on how these phenotypic changes occur is still unclear.

In this study, we sought to determine the long-term evolutionary adaption of C. neoformans when interacting with amoeba and whether the adaption affected virulence traits for animal hosts. Our results show that persistent amoeba predation was associated with the emergence of pleiotropic phenotypic changes of C. neoformans. 
137 Result

138 Selection of amoeba-resistance strains. We studied the interaction between C. neoformans and

139 Acanthamoeba castellanii by culturing them together on Sabouraud agar. For the initial

140 experiments, we used the well-studied common laboratory strain H99. The experimental setup

141 involved spreading approximately 200 cryptococcal cells on agar followed by placing

142 approximately $5000 \mathrm{~A}$. castellanii cells on the plate. After approximately month of co-

143 incubation, small colonies emerged within the predator zone of A. castellanii (Fig 1A),

144 sometimes under the mat of amoeba. Microscopic morphological analysis of cells in those

145 colonies revealed pseudohyphal and hyphal forms of C. neoformans (Fig 1B \& C). We selected

14620 single hyphal cells from two colonies (ten hyphae from each colony) and these were

147 transferred to a fresh Sabouraud agar plate without amoeba (Fig 1D, E \& I). After 24 h,

148 microcolonies composed exclusively of yeast cells emerged on the agar (Fig 1F \& J), which

149 manifested two distinct colonies morphologies, smooth and serrated, after two days of agar

growth (Fig 1G \& K). All of the cells from these colonies were yeasts (Fig 1H \& L). The same

151 experiment was then repeated with two environmental avirulent $C$. neoformans strains, A1-35-8

152 and Ftc555-1, but this time total 20 single hyphae were picked from four survival colonies (five

153 hyphae from each colony) to a fresh agar plate. Like the experience with H99, these strains

154 responded to the presence of amoeba by generating cells that formed colonies with various

155 cellular and colony morphologies, of which some (A4-6) were slightly serrated with

156 pseudohyphal cells (Fig 1M). We also observed some hyphal colonies formed by Ftc555-1 cells

157 but eventually they converted back to yeast cells when streaked on fresh agar medium (Fig 1N).

158 The results showed that after interacting with amoebae, C. neoformans can develop high variety

159 of cellular and colony morphologies even in amoebae-free medium. 
Six colonies from each strain were selected together with three controls, which were colonies on the same plate with isolates but without interacting with amoeba, for further phenotypic characterization (Fig 1D). These will be referred heretofore to as amoeba-passaged isolates with numbers preceded by the letters $\mathrm{H}, \mathrm{A}$, and $\mathrm{F}$ to indicate their origin from strains $\mathrm{H} 99, \mathrm{~A} 1-35-8$ and Ftc555-1, respectively. Controls will be referred to as C1-3 and ancestor will be referred to as A. To test if amoeba exerts selection pressure that resulted in amoeba-resistant cells, we examined if those isolates increased their survival during amoeba interaction. Isolates were then co-incubated with amoeba in the agar medium again, with $C$. neoformans in a cross, and amoeba were spotted in the center (Fig 2A). The radii of clear zones were measured as a function of time and these represented how well the amoeba clears the culture of $C$. neoformans. All of the amoeba-passaged isolates derived from $\mathrm{H} 99$ had reduced size of predator zone, when compared with their controls and ancestor strain (Fig 2B). In particular, the isolates that formed smooth colonies (H13, H16, H17) had the smallest predation zone (Fig 2B). This result implies that amoeba passage resulted in $C$. neoformans strains with increased ability to subsequently resist predation by amoeba. Next, we investigated the mechanism of the resistance. Samples were taken at the edge of the predator zone at the early stage of the interaction (week one), and observed under microscope. Isolates H13, H16 and H17 formed pseudohyphae while most of the cells in isolates H1, H2, H14 were in yeast form, with some displaying pseudohyphae (Fig 2C). However, no pseudohyphae were found in cells from controls and H99 ancestor colonies although pseudohyphae were formed eventually at the late stage of the interaction. Samples were also taken at a distance from the predation zone where cryptococcal cells had no contact with amoebae and in each of these regions all cells were in yeast form (Fig 2D). These results showed that pseudohyphal cells emerged rapidly from each of the amoeba-passaged strains even thought 
183 their cells were yeast prior to the incubation with amoebae and that pseudohyphal formation is a major mechanism of increased ability to resist predation.

185 When the isolates derived from A1-35-8 and Ftc555-1 strains were again exposed to $A$. castellanii, some but not all exhibited increased resistance to amoebae (Fig 3A \& B). Isolates

187 derived from A1-35-8 (A4-6) were significantly more resistant than the others (Fig 3A). That may be due to maintenance of pseudohyphal cell morphology by isolates A4-6 even in the amoebae-free medium. Isolates F3-5 manifested increased resistance to amoeba but unlike the H99 derived isolates, displayed no pseudohyphal formation but had larger cells when compared to their ancestor (Fig 3C \& D) at the early stage of interaction, which may be another survival

192 strategy for C. neoformans against amoebae. In this regard, phagocytosis of C. neoformans by macrophage was reduced by cell enlargement of $C$. neoformans [49-51]. The resistance of isolates F3-F5 to amoebae may reflect their larger cell size. factors that promote its pathogenicity, including formation and enlargement of a polysaccharide capsule, melanin production, extracellular secretion of urease, and cell enlargement. To evaluate whether the emergence of variant form of $C$. neoformans was accompanied by changes to known 199 virulence factors, we analyzed the virulence-related phenotypic characteristics of the isolates derived from the three strains. Isolates H13, H16 and H17 had larger capsule thickness relative to their ancestor when cultured in minimal medium but cell sizes were similar (Fig 4A and 5A). All of the isolates also had increased urease activity in comparison to their ancestor (Fig 6). Isolates $\mathrm{H} 1, \mathrm{H} 2$ and H14 manifested less melanin production (Fig 7). We also examined if there were any changes of virulence factors in isolates of environmental strains. Each of the isolates derived 
urease activity when comparing to their ancestor, but there was no change in cell size (Fig 4B, 5B and 6). Isolates F3-F6 has increased both their capsule and cell size in minimal medium (Fig 4C and 5C). They were also having $15-18 \%$ of cells with size larger than $10 \mu \mathrm{m}$ inside macrophages (Fig 5E) while approximately $80 \%$ of cells with larger than $10 \mu \mathrm{m}$ in macrophage medium in $37^{\circ} \mathrm{C} 9.5 \% \mathrm{CO}_{2}$ (Fig 5D). Moreover, all of the isolates of Ftc555-1 strain had increased urease activity, but reduced melanin production (Fig 6 and 7).

We further characterized the isolates in stress conditions by analyzing their growth under thermal stress and exposure to the antifungal drug fluconazole (Fig 8). Isolates H13, H16, H17 had reduced growth at $40^{\circ} \mathrm{C}$ and in the presence of fluconazole while $\mathrm{H} 1, \mathrm{H} 2$ and $\mathrm{H} 14$ had slightly increased their resistance to fluconazole compared to their ancestral strain. Isolates A4-A6 and F3-F6 displayed defects in growth at high temperature and after exposure to fluconazole.

Overall, the data show that the phenotypic changes were broad and diverse among isolates.

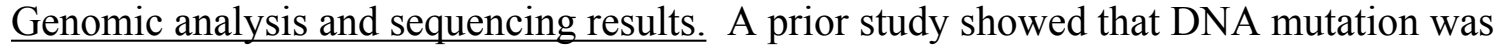
involved in pseudohyphal formation during amoebae interaction [52]. To find out if there are any such mutations or any other mutations in our experiments, the genomes of all isolates were sequenced. SNPs and indels were identified compared to the H99 reference genome (Table 1, 2 and table in S1 table). Genome sequencing revealed that $\mathrm{H}$ and $\mathrm{A}$ isolates acquired only a small number of SNPs and indels during amoeba passage, whereas F isolates acquired an order of magnitude more SNPs and indels. Two SNPs were identified in H1, H2, H14 in comparison with their ancestral strain H99. One of the SNPs is a missense mutation (M484R) in a gene encoding an oligopeptide transporter (CNAG_03013; OPT1). This mutation creates the replacement of methionine 484 with arginine. Opt1 has been shown to be required for transporting Qsp1, a quorum sensing peptide, into the receiving cells [53]. Another SNP is an intron variant in a gene 
encoding a protein kinase $\left(\mathrm{CNAG} \_2531 ; C P K 2\right)$ as part of the MAPK protein kinase family. Loss of CPK2 reduces melanin production in Niger seed media. For A1-35-8 derived isolates, a total of four SNPs were identified relative to the ancestral strain A1-35-8. In A1, one missense SNP was found in CNAG_01101, which encodes a hypothetical protein with a centrosomin Nterminal motif and also a single nucleotide deletion was identified in CNAG_03013, causing a frameshift at P358. Two SNPs were identified in A2 and A3 isolates, with one SNP leading to nonsense mutation in CNAG_03013 and another SNP resulting in missense mutation in CNAG_02858 which encodes adenylsuccinate synthetase. Another SNP in the A2 isolate was found in an intergenic region, a site with a high fraction of ambiguous calls. Isolates A4-6 had a single nucleotide deletion at gene CNAG_03622 (TAO3) and leads to the frameshift at residue 150 of 2392. This mutation is consistent to the finding in previous study that TAO3 mutation lead to the pseudohyphal phenotype [52]. In contrast to the A1-35-8 isolates, the rate of mutations in the Ftc555-1 isolates was 10 times higher, ranging from 22 to 77 SNPs (total 225 SNPs) and 715 indels (total 34 indels) in these isolates. Among those SNPs, three SNPs were annotated as high impact mutations resulting in disruption of the coding region (early stop codons and splice site mutations). One SNP results in a nonsense mutation (G407*) in CNAG_00570 which encodes Pkr1 (AMP-dependent protein kinase regulator) in F5 and F6 isolates. In addition, F3 and F4 isolates carry a single nucleotide deletion in CNAG_00570 leading to frameshift of residues 194 of $482 . \mathrm{Pkr} 1$ is one of the important components of cAMP/PKA pathway and negatively regulates Pka activity which is involved in morphogenesis, nutrient acquisition, stress responses and virulence in C. neoformans (Choi et al., 2012). Another SNP in the F1 isolate is a splice site mutation in CNAG_03013. In summary, there are three noteworthy observations in the sequence data: 1. The gene CNAG_03013 (OPT1) was impacted by non-synonymous SNP 
252 changes in all three strain backgrounds; 2. The previously described TAO3 mutation responsible

253 for pseudohyphal or hyphal formation was found in our isolates A4, A5, A6 [52]; and, 3. No

254 SNPs and indels were found in some of the isolates including H13, H16, H17 suggesting that the

255 phenotypic changes observed did not originate from single nucleotide variants in the genome.

256 Table 1. High and moderate impact SNPs found in passaged isolates

\begin{tabular}{|c|c|c|c|c|c|c|c|}
\hline Isolates & Chr & Position & Reference & Alternate & Gene ID & Gene function & $\begin{array}{l}\text { Effect of } \\
\text { mutation }\end{array}$ \\
\hline $\begin{array}{l}\mathrm{H} 1, \mathrm{H} 2, \\
\mathrm{H} 14\end{array}$ & 3 & 211613 & $T$ & G & CNAG_03013 & $\begin{array}{l}\text { Oligopeptide } \\
\text { transporter }\end{array}$ & M484R \\
\hline $\begin{array}{l}\mathrm{H} 1, \mathrm{H} 2, \\
\mathrm{H} 14\end{array}$ & 6 & 68953 & C & A & CNAG_02531 & $\begin{array}{l}\text { Calcium- } \\
\text { dependent } \\
\text { protein kinase }\end{array}$ & $\begin{array}{l}\text { Intron } \\
\text { variant }\end{array}$ \\
\hline A1 & 5 & 1208219 & $T$ & $C$ & CNAG_01101 & $\begin{array}{l}\text { Hypothetical } \\
\text { protein }\end{array}$ & R478G \\
\hline $\mathrm{A} 2, \mathrm{~A} 3$ & 3 & 213165 & G & $A$ & CNAG_03013 & $\begin{array}{l}\text { Oligopeptide } \\
\text { transporter }\end{array}$ & $\begin{array}{l}\text { Nonsense } \\
\text { mutation } \\
\text { W932* }\end{array}$ \\
\hline $\mathrm{A} 2, \mathrm{~A} 3$ & 3 & 594765 & A & G & CNAG_02858 & $\begin{array}{l}\text { Adenylsuccinate } \\
\text { synthetase }\end{array}$ & I346V \\
\hline $\mathrm{A} 2$ & 13 & 592173 & C & $\mathrm{T}$ & & $\begin{array}{l}\text { Intergenic } \\
\text { region }\end{array}$ & \\
\hline $\mathrm{F}^{+}$ & 3 & 213566 & G & $T$ & CNAG_03013 & $\begin{array}{l}\text { Oligopeptide } \\
\text { transporter }\end{array}$ & $\begin{array}{l}\text { Splice site } \\
\text { mutation }\end{array}$ \\
\hline $\mathrm{F} 5, \mathrm{F6}^{+}$ & 1 & 1469244 & $C$ & A & CNAG_00570 & $\begin{array}{l}\text { cAMP- } \\
\text { dependent } \\
\text { protein kinase } \\
\text { regulator }\end{array}$ & $\begin{array}{l}\text { Nonsense } \\
\text { mutation } \\
\text { G407* }\end{array}$ \\
\hline $\mathrm{FC}^{+}$ & 11 & 136455 & $T$ & G & CNAG_01506 & $\begin{array}{l}\text { Hypothetical } \\
\text { protein }\end{array}$ & $\begin{array}{l}\text { Splice site } \\
\text { mutation }\end{array}$ \\
\hline
\end{tabular}

$257{ }^{+}$Only high impact mutations of Ftc555-1 variants were shown in this table.

258 Table 2 High impact indels found in passaged A1-35-8 isolates

\begin{tabular}{|l|l|l|l|l|l|l|l|}
\hline Isolates & Chr & Position & Reference & Alternate & Gene ID & Gene function & $\begin{array}{l}\text { Effect of } \\
\text { mutation }\end{array}$ \\
\hline A1 & 3 & 211137 & GC & G & CNAG_03013 & $\begin{array}{l}\text { Oligopeptide } \\
\text { transporter }\end{array}$ & $\begin{array}{l}\text { Frameshift at } \\
\text { P358 }\end{array}$ \\
\hline $\begin{array}{l}\text { A4, A5, } \\
\text { A6 }\end{array}$ & 2 & 363200 & CA & C & CNAG_03622 & Cell polarity & $\begin{array}{l}\text { Frameshift at } \\
\text { N150 }\end{array}$ \\
\hline
\end{tabular}


To determine if the high impact mutations we identified in genes PKR1, OPT1, CNAG_02531 and CNAG_01506 are responsible for resistance to the killing of amoebae, deletion mutants of the candidate genes in the H99 background were co-incubated with amoebae on solid medium. However, the predator zones from these mutants were comparable with the parental strain (S2 Fig).

Aneuploidy. We next hypothesized that emergence of aneuploidy could be a source of evolutionary adaptation because aneuploidies are frequent in C. neoformans and it has been shown to play crucial roles in stress resistance $[54,55]$. To this end, the chromosomal copy numbers of the isolates were defined based on the normalized depth of sequence coverage. The analysis revealed that there were duplications of chromosome 8 in isolates H13, H16 and H17, but no chromosomal duplication has been found in other isolates (Fig 9A). The results were confirmed by qPCR with two selected isolates, H14 and H17 (Fig 9B and S1 Fig). We next investigated if this chromosomal duplication was responsible for the pseudohyphal formation and other phenotypic changes. In order to do so, H17 was passaged in fresh rich medium every

274 day for 30 days to eliminate the duplication. The elimination was confirmed by qPCR (Fig 9C). H17 euploid strain $\left(\mathrm{H} 17^{\mathrm{eu}}\right)$ was then co-incubated with amoebae culture in solid medium, and

276 samples were taken from the edge of the predation zone and visualized under microscope. No

277 pseudohyphae could be observed in H17 eu (Fig 9D). In such case, the observation was similar to

278 what we found in $\mathrm{H} 99$, but distinct from $\mathrm{H} 17$ aneuploid strain $\left(\mathrm{H} 17^{\text {aneu}}\right)$ that forms mostly

279 pseudohyphae after one-week co-incubation (Fig 9D). Not surprisingly, H1 $7^{\text {eu }}$ had decreased ability for amoebal resistance, having a similar size of the predator zone as H99 while H17 $7^{\text {aneu }}$

281 had a smaller predator zone (Fig 9E). The capsule size of H1 $7^{\text {eu }}$ was smaller than $\mathrm{H} 17^{\text {aneu }}$ and 282 similar to $\mathrm{H} 99$, suggesting that the duplication of chromosome 8 results in larger capsule size 
283 (Fig 9F). H17 eu had lower urease activity than H17 aneu but comparable level with H99 after $1 \mathrm{~h}$

284 (Fig 9G). However, the urease activity of H17 eu increased faster than that of H99 after $1.5 \mathrm{~h}$.

285 The result implied that the chromosomal duplication may be responsible partially the high urease 286 activity found in $\mathrm{H} 17^{\text {aneu }}$.

Aneuploidy can arise from multinucleate state through transient polyploidization after failed cytokinesis or cell fusion. The filamentous multinucleate fungus Ashbya gosypii exhibits both polyploidy and aneuploidy frequently after cell division [56]. Since pseudohyphae have a cytokinesis defect and multinuclei within a common cytosol, we asked if the pseudohyphal formation may lead to ploidy variation and thus may become one of the sources of phenotypic variation. Consequently, H99 expressing green fluorescent protein-labeled histione-2 (GFP-H2B) were visualized by time-lapsed imaging, and a nucleus fusion have been observed in one of the pseudohyphae after nuclei separation (Fig $10 \&$ S1 Movie). This event provides evidence that polyploidization can exist in pseudohyphae and thus may have a high chance of leading to aneuploidy and phenotypic variation.

Epigenetic modifications. Chromatin remodeling can rapidly moderate transcriptional response in order to let the microorganism to adapt rapidly to stressful conditions in hosts. Histone

299 acetyltransferase activity has been shown to be essential for $C$. neoformans in virulence regulation and response to host environments [57]. Since the phenotypes changed rapidly after $C$. neoformans interacted with amoebae, we hypothesized that histone acetylation may be involved in the phenotypic changes. To determine whether the evolution of $C$. neoformans after interacting with amoebae also involved histone modification, we compare the quantitation of the 

damages to their host cells. 
growth curves of the three $C$. neoformans strains. Two growth curves were prepared in similar conditions, except the initial number of yeasts. Starting the curve with a high concentration of cells $\left(1.0 \times 10^{7}\right)$ displayed a similar growth increase for F5 and F6 cells during the first 36 hours, but the very opposite was observed when the curve started with a low concentration of cells $\left(5.0 \times 10^{3}\right)$, with a higher growth increase for Ftc555-1 strain (S4 Fig). Hence, all three strains were able to establish themselves in mice initially and survive clearance by innate immunity but the F5 and F6 were subsequently cleared, presumably by the development of acquired immunity.

To gain more insight into the immune responses elicited by Ftc555-1, F5 and F6 in the lung we studied several cytokine responses. In the lungs, the levels of pro-inflammatory cytokines, including tumor necrosis factor alpha (TNF- $\alpha$ ) and interferon gamma (IFN- $\gamma$ ), were increased and remained high after the $60 \mathrm{~d}$ post-infection with F5 and F6 strains (Fig 12). The lung cytokine response shows that F5 and F6 elicit lower levels of IL-10 than Ftc555-1, which could help their clearance from lung tissue relative to Ftc555-1 since reduction of this anti-inflammatory cytokine has been associated with increased resistance to cryptococcal infection in mice [59]. Interestingly, the levels of the same molecules were different when we analyzed the systemic immune response as measured by cytokines in their spleens (S5 Fig). These results may indicate that sustaining high levels of these cytokines may stimulate an inflammatory reaction, which could be associated with resolution of the infection of the mutant strains. These cytokine results show that Ftc555-1 is eliciting quantitatively different immune responses from the parent strain Ftc555-1 consistent with the notion that the differences in virulence observed for these strains reflect differences in the effectiveness of the immune responses triggered.

We also examine the virulence of the isolates using wax moth larvae model, and isolates H13, H16 and H17 were less virulence than their ancestor (S6A Fig). This may due to the fact 

available under aCC-BY 4.0 International license.

352 that these isolates can form pseudohyphae rapidly in the larvae, and pseudohyphal $C$.

353 neoformans are attenuated for virulence in wax moth larvae [52]. However, so far, there was no

354 statistically significant increased virulence of isolates in the wax moth larvae infection model (S6

355 Fig).

356

357 


\section{Discussion}

359

360

361

362

363

364

365

366

367

368

369

370

371

372

373

374

375

376

377

378

379

380

In the past two decades, the concept that amoeba acts as selective pressure for virulence traits of environmental microbes has gained considerable traction. For fungal pathogens, concordance between virulence factor function in amoeba and macrophages has been demonstrated for $C$. neoformans [14,40], Aspergillus fumigatus [23,60] and Paracoccidioides spp. [24], but many questions remain on how fungal-protozoal interactions select for mammalian virulence. In this study, we investigated how interactions with amoebae affected the phenotype and genotype of $C$. neoformans to explore the mechanisms behind this long-term evolutionary adaptation. Our results provide new insights on how amoeba predation can drive the evolution of $C$. neoformans since survivors emerge that show major phenotypic and genetic differences from the founder strain. The phenotypic diversity may facilitate $C$. neoformans adaptation to different hosts and thus enhance its virulence.

Pseudohyphae formation was the most common response to $C$. neoformans survival when faced with amoeba predation. This result confirms an older observation that pseudohyphal formation was a 'escape hatch' for $C$. neoformans survival when preyed upon by amoebae [61]. Different fungal morphologies are reported to trigger different killing mechanisms by amoeba [62] and the C. neoformans filamentous form may be more resistant to killing. Similar to our observation, Nielson et al [61] reported that when C. neoformans was co-cultured with amoebae, most of the fungal cells were killed with survivors forming colonies that contained pseudohyphae. Most of their isolates remained pseudohyphal, with only one out of eight isolates reverting back to the yeast form. That result differed from ours, since most of the pseudohyphal isolates in this study reverted to yeast forms after removal from the amoebae culture, such that only 3 of 18 isolates studied in detail maintained a stable pseudohyphal phenotype. Those three isolates (A4 - A6) 
381 have a single nucleotide deletion in TAO3 gene shown in whole genomic sequencing, and consistent to mutations in RAM/MOR pathway ofthe pseudohyphal variants reported in the previous study [52].

384 Previous studies have focused primarily on cryptococcal isolates with pseudohyphae phenotypes 385 derived from amoeba, but in this study, we investigated in detail those amoeba-resistant isolates with unstable pseudohyphal phenotypes. We found that although some of the isolates (H13. H16, H17) reverted to yeast, they were able to form pseudohyphae quicker than their parental strain when they were exposed to amoebae again. These isolates were less virulent in Galleria infection model, a finding consistent with prior reports that the pseudohyphae strains were less virulent in animal models. Interaction with amoebae also resulted in measurable virulencerelated phenotypic changes in $C$. neoformans, confirming that amoebae can play a powerful role

392 in the selection of virulence factors, which are related to the pathogenesis of human disease. Of note, we selected only six isolates from each strain for further characterization, but all of them

394 had changes, suggesting that the microevolution occurs frequently and rapidly when exposed to 395 amoebae. Moreover, the changes were pleiotropic and included differences in colony morphology, capsule size, cell size, urease activity, melanin production, susceptibility to thermal

397 stress and an antifungal drug. However, isolates studied revealed a different configuration of 398 phenotypic changes although they tended to cluster in groups from the same survival 399 pseudohyphal colony (S7 Fig). Overall, the interaction of C. neoformans with amoebae400 passaged isolates with increased phenotype diversity. Since there are many types of amoeboid 401 predators in the soil and C. neoformans does not know the identity of the phagocytic predator, 402 generating great diversity in strains could provide this fungus with insurance that some will 
403

404

405

406

407

408

409

410

411

412

413

414

415

416

417

418

419

420

421

422

423

424

425

survive. Hence, the diversity observed among isolates that survived amoeba predation suggests a bet hedging strategy for survival based on the generation of phenotypic diversity.

To identify the mechanism for the phenotypic changes, we compared the whole genome sequencing of isolates and ancestral strains using deep sequencing to identify point mutations, amplification or deletion of chromosomal segments and whole-chromosome aneuploidy. We found that there were only two SNPs in H99 derived isolates, four SNPs and two indels in the A1-35-8 derived isolates. Isolates from the same survival pseudohyphal colonies had similar SNPs, which is consistent with the similarity of their phenotypic changes, suggesting that the point mutations may be associated with some of the phenotypic changes. Interestingly, there were total 252 SNPs in Ftc555-1 derived isolates with an average of 48 SNPs among isolates (range of 22-80), a rate approximately10 times higher than $\mathrm{H} 99$ and A1-35-8. That may be explained by the fact that the ancestral Ftc555-1 strain contains a splice donor site mutation in $M L H 1$, a gene involved in mismatch repair of nuclear DNA. This predicted high impact, loss of function mutation ( $\mathrm{G}$ to A change at position 1270268 of chr 6 ) is also found in all sequenced Ftc555-1 progeny isolates. Since the Idnurm laboratory has reported that the loss of $M L H 1$ results in elevated mutation rates [63], Ftc555-1 is likely to be a hypermutator strain. Increased mutation rates will drive phenotypic variations and some of those may be adaptive for survival in stressful environments, leading to rapid microevolution. On the other hand, the sequencing revealed that one gene (CNAG_03013; OPT1) was impacted by non-synonymous SNP changes and single nucleotide deletion in all three strain backgrounds. OPT1 has been identified by Madhani group as an oligopeptide transporter required for transporting Qsp1, a quorum sensing peptide, into the receiving cells [53]. Deletion of OPT1 exhibits similar phenotypes to our isolates, including increased capsule size and reduced melanin production, suggesting that this 
mutation may cause some of the phenotypic changes in our isolates. By reviewing the published sequences of 387 clinical and environments strains [64], we found that 6 of 287 clinical isolates contains high impact, potential loss of function mutations in OPT1, but no OPT1 mutations in those 100 environmental isolates. The Fraser laboratory also reported that one of the clinical isolates in their study contains an inversion in chromosome 3 and affect two genes while one of them are $O P T 1$ [65]. The relatively high frequency of mutations in $O P T 1$ among clinical isolates suggests that this gene may be under particular selection during human infection. Another interesting gene mutation found in Ftc555-1 isolates was in the gene PKRl. This was a high impact mutation in F3, F4, F5 and F6, which exhibited phenotypes of titan cells and enlarged capsules inside macrophages and in macrophage medium. Pkr1 is known to be a negative regulator of titan cells and capsule enlargements in laboratory strains and clinical isolates $[58,66]$. A pkrl deletion mutant exhibit both enlarged capsule and titan cell production. It is also hypervirulent in a murine infection model [58].

The relatively low number of SNPs raises the question on how some of these strains change rapidly in response to amoeba predation that result in such broad and rapid phenotypic changes. Therefore, we also investigated the impact of whole-chromosome aneuploidy on isolates. An extra copy of chromosome 8 has been found in three isolates (H13, H16 and H17) which were isolated from the same pseudohyphal survival colony. Aneuploidy is caused by abnormal chromosomal segregation and can happen within even a single mitotic division, so this type of mutant can occur rapidly. This drastic DNA structural change often results in decreased fitness [67]. However, when fungi are exposed to stress, such as antifungal drugs, specific chromosomal aneuploidies can be advantageous through selection for increased gene expression of a subset of 
genes [55,68-72]. In C. albicans and C. neoformans, extra copies of specific chromosome containing drug resistance genes have been frequently found in antifungal drug resistance strains $[55,70,71]$. Likewise, $C$. neoformans could gain an extra chromosome as a solution for adaptation when the fungi encounter threats from amoebae. For instance, chromosome 8 contains one gene $(Z N F 2)$ which encodes a zinc-finger transcription factor that drives hyphal growth upon overexpression [73]. Chromosome 8 also contains another gene $(C B K 1)$ that is responsible for pseudohyphal formation [52,74]. $C B K 1$ encodes serine/threonine protein kinase which is one of the components of RAM pathway. Mutants in the RAM pathway have pseudohyphal phenotype, but we are not aware of any reports showing the effect of the overexpression of $C B K 1$ on pseudohyphae morphology. Since filamentous morphologies are important for resistance to phagocytosis by amoebae, it is possible that duplication of chromosome 8 could increase the cryptococcal fitness rapidly after exposure to amoebae. Indeed, when we re-introduced those aneuploid strains to amoebae, they could switch to filamentous forms quicker than their ancestor and efficiently resisted killing by amoebae. When we eliminated the chromosomal duplication, the phenotypes were restored back to wildtype level, supporting that there is strong link between duplication of chromosome 8 and amoebae resistance and other changes on virulence phenotypes such as capsule size and urease activity. In addition, there is no point mutations or structural changes such as amplification or deletion of chromosomal segments in these isolates. Therefore, aneuploidy may be the major source of the phenotypic change in that particular group of isolates.

Pseudohyphae are chains of elongated yeast cells that are unable to undergo cytokinesis completely, leading to multinuclei. Multinucleated cells showed a high level of chromosome instability, resulting in polyploidy and aneuploidy in eukaryotic cells [56]. Previous study of 
472 live-cell imaging on Candida albicans showed that hyphal cells occasionally generated

473 multinucleated yeast cells [75], with polyploidy and/or aneuploidy, but there are very limited

474 studies on whether pseudohyphal or hyphal formation may directly affect the ploidy variation. In

475 this study, nuclear division, detected with GFP-H2B, was observed in cryptococcal

476 pseudohyphae isolated from amoebae culture. The time-lapse imaging detected a nuclear fusion

477 event, suggesting the cell experienced atypical nuclear division and potentially may undergo

478 polyploidization which frequently generates their offspring with amplification of chromosomal

479 segments or whole-chromosome aneuploidy. This result implies that interaction with amoebae

480 not only contributes to the selection and maintenance of traits in C. neoformans, but also may

481 drive heritable variation through pseudohyphae formation.

The 'amoeboid predator-fungal animal virulence hypothesis' formulates the notion that the capacity for virulence in soil fungi with no need for an animal host arose accidently from the traits for survival against ameboid predators that accidently also functioned as virulence factors

486 for animal infection [12]. Consistent with this notion there is a remarkable concordance between

487 fungal phenotypes that promote survival against amoeba and in animal hosts [14,23] and passage

488 in amoeba is associated with increased virulence for several fungal species $[24,39,76]$. Analysis

489 of virulence for the amoeba-selected strains described in our study in wax moths revealed no

490 major changes in virulence from the parental strains. It is possible that this host does not

491 discriminate between passaged and non-passaged $C$. neoformans cells or that none of the isolates

492 tested gained or lost traits associated with virulence in that particular host. It is also possible that

493 these strains already had the maximum pathogenic potential [77] for these animal hosts, which

494 could not be further increased by amoeba interactions. However, we did observe that some 
amoeba-passaged strains were significantly more cytotoxic for macrophages in vitro. This result is consistent to the finding that which those strains also had great resistance to amoebae killing. The mechanism behind that is still unclear. However, those particular amoeba-passage strains can form larger cell size and capsule in both amoebae and macrophage culture and that may help them to escape from and cause damages to the host cells. These results fit the theory that amoebae are the training grounds for macrophage resistance of pathogens since the hostile environments in amoebae and macrophage are similar. Among these strains, the virulence of isolates F5 and F6 were further tested in murine infection model. These particular strains were picked because they acquired a mutation in $P K R l$, and deletion of $P K R l$ has been shown to increase virulence [58]. However, neither F5 and F6 exhibited hypervirulence phenotype during murine infection, and instead were cleared faster than their parental isolate. It is noteworthy that the nonsense mutation found in F5 and F6 located in codon 407 which is only 75 codons prior to the original stop codon of $P K R 1$. It is possible that the mutation results in altered function rather than loss of function and this is not sufficient to reproduce the hypovirulence phenotype caused by full PKRl knockout. Microbial virulence is a complex property that is expressed only in a susceptible host and host damage can come from the microbe or the immune response. Both F5 and F6 were able to establish themselves in the lung but triggered a more effective immune responses that cleared them. This finding implies the occurrence of other amoeba-selected changes that affect the immune response including overriding of the hypervirulence phenotype caused from the mutation of $P K R l$ by compensation from other mutations or changes.

The amoeba-passaged $C$. neoformans selected in our study differ from those reported in prior studies $[24,39,76]$ in that they did not increase in virulence. Instead, we observed reductions in murine virulence for two of the isolates studied despite increased capacity to damage 
macrophages from their long interaction with amoeba. Given the pleiotropic changes observed in our isolate set it is possible that we did not sample sufficient numbers to observed more virulent strains. Our study differs from prior amoeba-C. neoformans studies [39] in that it involved

521 prolonged selection on a semi-solid agar surface in conditions that favored the protozoal cells by

522 the presence of cations. In these conditions, amoeba dominance is manifested by a zone of

523 fungal growth clearance where only occasional $C$. neoformans colonies emerged after several

524 weeks. These colonies presumably emerged from resistant cells that survived the initial amoeba

525 onslaught and gave rise to the variant strains that were analyzed in this study. We posit that

526 these amoeba-resistant cells were very rare in the parent $C$. neoformans population and had

527 emerged from the mechanisms discussed above, namely mutation and aneuploidy, which by

528 chance conferred upon those cells amoeba resistance. Alternatively, those colony ancestor cells

529 represent rare cells that were able to sense the amoeba danger and turn on diversity generating

530 mechanisms that occasionally produced amoeba-resistant strains. In this regard, C. neoformans

531 can sense amoeba and respond by increasing the size of its capsule by sensing protozoal

532 phospholipids [40] but this process takes time and fungal cell survival probably depends on the

533 race between adaptation and predation. The selection versus adaptation explanations for the

534 origin of these are not mutually exclusive and both could have been operational in these

535 experiments. These survivor cells then grew into a colony under constant amoeba selection

536 where they gave rise to progeny cells where these phenotypic diversity generating mechanisms

537 were maintained and amplified thus accounting for the phenotypic diversity observed in this 538 study. 
540 In summary, amoebae predation places great selective pressure in C. neoformans resulting in the

541 rapid emergence of new phenotypes. The mechanism for these changes includes mutations and

542 aneuploidy, which combine to create great phenotypic diversity. The effect of the phenotype

543 diversification on the fitness of the fungi vary within the same or different hosts, which could

544 promote fungal survival by a bet-hedging strategy that spreads the risk in situations where the

545 environmental threat is unpredictable. Given that human infection also results in rapid fungal

546 microevolution in this host, it is likely that similar mechanisms occur in vivo when this fungus

547 comes under attack by immune cells. Indeed, several studies have shown microevolution of

548 Cryptococcus during mammalian infection [65,78-80]. A bet hedging strategy that generates a

549 prodigious number of phenotypes would increase survival in the face of unknown threats and

550 could represent a general mechanism for survival in soils. Interference with the mechanism

551 responsible for generating this plasticity could in turn result in new antimicrobial strategies that

552 would reduce the emergence of diversity and thus simplify the problem for the immune response.

553 Hence, it is interesting to hypothesize that amoeba predation in C. neoformans pushes a trigger

554 that sets forth a series of events that generate diversity and similar mechanisms exist in other soil

555 fungi that must routinely confront similar stresses. 


\section{Method and material}

558

559

560

561

562

563

564

565

566

567

568

569

570

571

572

573

574

575

576

577

578
Ethics statement. All animal procedures were performed with prior approval from Johns Hopkins University (JHU) Animal Care and Use Committee (IACUC), under approved protocol numbers $\mathrm{MO} 18 \mathrm{H} 152$. Mice were handled and euthanized with $\mathrm{CO}_{2}$ in an appropriate chamber followed by thoracotomy as a secondary means of death in accordance with guidelines on Euthanasia of the American Veterinary Medical Association. JHU is accredited by AAALAC International, in compliance with Animal Welfare Act regulations and Public Health Service (PHS) Policy, and has a PHS Approved Animal Welfare Assurance with the NIH Office of Laboratory Animal Welfare. JHU Animal Welfare Assurance Number is D16-00173 (A327201). JHU utilizes the United States Government laws and policies for the utilization and care of vertebrate animals used in testing, research and training guidelines for appropriate animal use in a research and teaching setting.

Cell culture. Acanthamoeba castellanii strain 30234 was obtained from the American Type Culture Collection (ATCC). Cultures were clinical isolate of maintained in PYG broth (ATCC medium 712) at $25^{\circ} \mathrm{C}$ according to instructions from ATCC. C. neoformans var. grubii serotype A strain H99 and two environmental isolates A1-35-8 and Ftc555-1 were used for the interaction with amoebae, and these strains were originally obtained from John Perfect (Durham, NC). Both A1-35-8 and Ftc555-1 are environmental strains. A1-35-8 with genotype of VN1 molecular type is isolated from pigeon guano in US while Ftc555-1 is isolated from a mopane tree in Botseana and portrayed VNB molecular type. Both strains were avirulent in mouse model. Histone 2BGFP tagged (C1746) H99 strain which was used for visualization of nuclear division of pseudohyphae was obtained from Kyung Kwon-Chung (Bethesda, MD) [81]. Cryptococcal cells 
were cultivated in Sabouraud dextrose broth with shaking $(120 \mathrm{rpm})$ at $30^{\circ} \mathrm{C}$ overnight $(16 \mathrm{~h})$ prior to use in all experiments.

Bone-marrow derived macrophages (BMDM) were isolated from the marrow of hind leg bones of 5- to 8-wk-old C57BL-6 female mice (Jackson Laboratories, Bar Harbor, ME). For differentiation, cells were seeded in $100 \mathrm{~mm}$ TC-treated cell culture dishes (Corning, Corning, NY) in Dulbecco's Modified Eagle medium (DMEM; Corning) with 20 \% L-929 cellconditioned medium, 10 \% FBS (Atlanta Biologicals, Flowery Branch, GA), 2mM Glutamax (Gibco, Gaithersburg MD), 1 \% nonessential amino acid (Cellgro, Manassas, VA), 1 \% HEPES buffer (Corning), $1 \%$ penicillin-streptomycin (Corning) and $0.1 \%$ 2-mercaptoethanol (Gibco) for 6-7 days at $37{ }^{\circ} \mathrm{C}$ with $9.5 \% \mathrm{CO}_{2}$. Fresh media in $3 \mathrm{ml}$ were supplemented on day 3 and the medium were replaced on day 6. Differentiated BMDM were used for experiments within 5 days after completed differentiation.

Assay of $A$. castellanii and $\boldsymbol{C}$. neoformans interaction. Two hundred C. neoformans yeast cells were spread on Sabouraud agar, and incubated at $30^{\circ} \mathrm{C}$ overnight. A. castellanii in total $5 \times 10^{3}$ cells were dropped randomly at several locations on the agar plate containing C. neoformans.

Plates were sealed with parafilm and incubated at $25^{\circ} \mathrm{C}$ for $3-4$ months until survival colonies of C. neoformans emerged.

To isolate individual cell (hyphae or pseudohyphae in this case) out from the colony (Fig 1D), survival colonies were randomly picked from the plate to a $3 \mathrm{~cm}$ culture dish with PBS using pipette tips. Individual cells were picked under a light microscopy using pipette and transferred into a fresh Sabouraud agar. The plates were incubated at $30{ }^{\circ} \mathrm{C}$. After $24 \mathrm{~h}$ incubation, the morphologies of microcolony were visualized using a Zeiss Axiovert 200M inverted microscope with a $10 \times$ phase objective. After $72 \mathrm{~h}$ incubation, colony morphologies were examined using 
602

603

604

605

606

607

608

609

610

611

612

613

614

615

616

617

618

619

620

621

622

623

624

Olympus SZX9 microscope with 1x objective and 32x zoom range. Morphologies of cells from colonies were visualized using Olympus AX70 microscope with 20x objective using the QCapture Suite V2.46 software (QImaging, Surrey, Canada).

Amoebae killing assay. $C$. neoformans in $5 \times 10^{6}$ cells were spread as a cross onto Sabouraud agar, and incubated at $30{ }^{\circ} \mathrm{C}$ for overnight. A. castellanii $\left(10^{4}\right)$ cells were dropped at the center of the $C$. neoformans cross. The plates were sealed in parafilm, and incubated at $25{ }^{\circ} \mathrm{C}$. The distance from center to the edge of the clear predator zones in four directions was measured after 1-3 weeks incubation. The data were represented as the average of the distances of clear zone from four direction.

C. neoformans cells were also taken from the edge of the clear zone and at the end of the cross after 1- week incubation, and visualized using Olympus AX70 microscope with 20x objective. For samples of Ftc555-1 strains, the cells were counterstained with India ink.

Capsule and cell size. $C$. neoformans cells were incubated in minimal medium (15 mM dextrose, $10 \mathrm{mM} \mathrm{MgSO}$, $29.4 \mathrm{mM} \mathrm{KH}_{2} \mathrm{PO}_{4}, 13 \mathrm{mM}$ glycine, $3 \mu \mathrm{M}$ thiamine- $\mathrm{HCl}$ ) at $30{ }^{\circ} \mathrm{C}$ for $72 \mathrm{~h}$. In addition, Ftc555-1 and its isolates were incubated in medium for BMDM at $37{ }^{\circ} \mathrm{C}$ for 24 h. $\operatorname{BMDM}\left(1.5 \times 10^{6}\right.$ cells $)$ were also infected with Ftc555-1 and its isolates $\left(1.5 \times 10^{6}\right.$ cells $)$ in 6-well plates. After $24 \mathrm{~h}$ infection, the culture supernatant was collected and the plates were washed once to collect the extracellular C. neoformans. The intracellular $C$. neoformans was collected by lysing the host cell with sterile water. The cells were stained with $0.1 \%$ Uvitex $2 \mathrm{~B}$ (Polysciences, Warrington, PA) for $10 \mathrm{~min}$ and washed two times with PBS. The capsule was visualized by India ink negative staining by mixing cell samples with equal volume of India ink on glass slides and spreading the smear evenly with coverslips. The images with a minimum 100 randomly chosen cells was taken by using Olympus AX70 microscopy with 40x objective at 
625 bright-field and DAPI channel. The areas of cell body and whole cell (cell body plus capsule)

626

627

628

629

630

631

632

633

634

635

636

637

638

639

640

641 642 interval.

643

644

645

646

647 sample.

were measured using image $\mathrm{J}$ software. The capsule thickness was calculated by subtracting the diameter of whole cell from that of cell body. The cell size was presented as the diameter of cell body without capsule. Three biological independent experiments were performed for each

lactate dehydrogenase $(\mathrm{LDH})$ release assay. BMDM cells $\left(5 \times 10^{4}\right.$ cells/well $)$ were seeded in 96-well plates with BMDM for overnight. To initiate the phagocytosis, C. neoformans with $5 \times$ $10^{5}$ cells in the presence of $10 \mu \mathrm{g} / \mathrm{ml} 18 \mathrm{~B} 7 \mathrm{mAb}$ were added in each well of BMDM culture. The culture plates were centrifuged at $1200 \mathrm{rpm}$ for $1 \mathrm{~min}$ to settle yeast cells on the monolayer of macrophage culture. After $48 \mathrm{~h}$ infection, LDH release were assessed using CytoTox-ONE Homogeneous Membrane Integrity Assay kit (Promega, Madison, WI) according to the manufacturers' instructions.

Urease activity. C. neoformans in $10^{8}$ cells were incubated in $1 \mathrm{ml}$ of rapid urea broth (RUH) developed by Roberts [82] and adapted by Kwon-Chung [83] at $30^{\circ} \mathrm{C}$. After $1-4 \mathrm{~h}$ incubation, cells were collected by centrifugation and $100 \mu 1$ of supernatant were transferred to 96 -well plate. The absorbance of the supernatant was measured at $570 \mathrm{~nm}$ using EMax Plus microplate reader (Molecular Devices, San Jose, CA). The assay was performed in Triplicate for each time

Melanin quantification. $C$. neoformans in $10^{4}, 10^{5}, 10^{6}$ and $10^{7}$ cells were spotted on agar of minimal medium supplemented with $1 \mathrm{mM}$ L-DOPA (Sigma Aldrich, St Louis, MO). The plates were incubated at $30{ }^{\circ} \mathrm{C}$ without light. Photos were taken after 1-3-day incubation on a white light illuminator. The photos of samples were always taken together with their ancestors under the same condition in order to avoid different exposure time or light adjusted by the camera. The 
648 obtained photos were then converted to greyscale using image $\mathrm{J}$ software. The regions of the 649 colonies were selected and the pixels of each selected region were quantified in grayscale. The 650 relative grayscale of the colonies from samples were normalized by the grayscale of the colonies

651 of ancestors. The representation data shown in this paper are at the cell number of $10^{6}$ cells and

652 at the time point of $24 \mathrm{~h}$. Three biological independent experiments were performed for each 653 sample.

654 Macrophage killing assay. BMDM cells $\left(5 \times 10^{4}\right.$ cells/well $)$ were infected with $C$. neoformans $655\left(5 \times 10^{4}\right.$ cells $)$ in the presence of $10 \mu \mathrm{g} / \mathrm{ml} 18 \mathrm{~B} 7 \mathrm{mAb}$. The culture plates were centrifuged at $6561200 \mathrm{rpm}$ for $1 \mathrm{~min}$ to settle yeast cells on the monolayer of macrophage culture. After $24 \mathrm{~h}$ 657 infection, phagocytized cryptococcal cells were released by lysing the macrophages with 658 sterilized water. The lysates were serially diluted, plated onto Sabouraud agar and incubated at $65930{ }^{\circ} \mathrm{C}$ for $48 \mathrm{~h}$ for colony form unit (CFU) determination. This experiment was performed in 660 triplicates for each strain.

Virulence assay in Galleria mellonella. G. mellonella larvae were purchased from Vanderhorst Wholesale (Saint Mary's, OH). Larvae were picked based on weight (175 - $225 \mathrm{mg}$ ) and appearance (creamy white in color). Larvae were starved overnight at room temperature. Next day, overnight cultures of $C$. neoformans that grew in Sabouraud broth were washed three times with PBS and diluted to $1 \times 10^{5}$ cells $/ \mathrm{ml}$. Cells in $10 \mu 1$ were injected into the larva via the second last left proleg paw with $31 \mathrm{G}$ needles. Infected larvae were incubated at $30^{\circ} \mathrm{C}$ and the number of

667 death larvae were scored daily until all the larvae infected with $C$. neoformans ancestral strains in 668 this study were dead. Control groups of larvae were inoculated with $10 \mu \mathrm{L}$ of sterile PBS. 
670 Whole genome sequencing and variant identification. Genomic DNA was prepared using

671 cetyltrimethylammonium bromide (CTAB) phenol-chloroform extraction as described

672 previously [84]. Genomic DNA was further purified using a PowerClean DNA cleanup kit

673 (QIAGEN, Hilden, Germany). Libraries were constructed using the Illumina DNA Flex Library

674 kit and were sequenced on an Illumina HiSeq2500 to generate paired 150 base reads. An average

675 of 145X sequence depth (range 69-176X) was generated for each sample. All sequence for this

676 project is available in NCBI under BioProject PRJNA640358.

677 Reads were aligned to the $C$. neoformans H99 assembly [85] using BWA mem v0.7.12 [86].

678 Variants were identified using GATK v3.7 [87]; HaplotypeCaller was invoked in GVCF mode with

679 ploidy $=1$, and genotypeGVCFs was used to predict variants in each strain. The workflow used to

680 execute these steps on Terra (terra.bio) is available on Github

681 (https://github.com/broadinstitute/fungal-

682 wdl/tree/master/workflows/fungal_variant_calling_gatk3.wdl). Sites were filtered with

683 variantFiltration using $\mathrm{QD}<2.0, \mathrm{FS}>60.0$, and $\mathrm{MQ}<40.0$. Genotypes were filtered if the

684 minimum genotype quality $<50$, percent alternate allele $<0.8$, or depth $<10$

685

(https://github.com/broadinstitute/broad-

686 fungalgroup/blob/master/scripts/SNPs/filterGatkGenotypes.py). Genomic variants were

annotated and the functional effect was predicted using SnpEff v4.1g [88].

Cryptococcal cell karyotyping. Cell karyotypes were analyzed by quantitative PCR. qPCR

primers used in this study have been published in Gerstein et al. 2015. qPCR reactions were 
693

694

695

696

697

698

699

700

701

702

703

704

705

706

707

708

709

710

711

712

713

714

715

primer, 10-ng genomic DNA from CTAB extraction, and distilled water (dH2O). Cycling conditions were $95^{\circ} \mathrm{C}$ for $5 \mathrm{~min}$ followed by 40 cycles of $95^{\circ} \mathrm{C}$ for $15 \mathrm{~s}$ and $55^{\circ} \mathrm{C}$ for $1 \mathrm{~min}$. Melt curve analysis was performed in $0.5^{\circ} \mathrm{C}$ increments from 55 to $95^{\circ} \mathrm{C}$ for $5 \mathrm{~s}$ for each step to verify that no primer dimers or product from misannealed primers had been amplified. Threshold cycle (CT) values were obtained using StepOnePlus software version 2.3 (Applied Bioscience) where the threshold was adjusted to be within the geometric (exponential) phase of the amplification curve. Chromosome copy numbers were determined using a modified version of the classical CT method as described by [69].

Visualization of nuclear division in pseudohyphae. Histone 2B-GFP tagged H99 (C1746) was interacted with $A$. castellanii on Sabouraud agar as described above until survival colonies with pseudohyphae emerged. The colonies were transferred on the well of $18 \mathrm{~B} 7 \mathrm{Ab}$ coated coverslip bottom MatTek petri dishes with 14mm microwell (MatTek Brand Corporation, Ashland, MA) in minimal medium. After $30 \mathrm{~min}$ incubation to allow for settling down the cells, $2 \mathrm{ml}$ of minimal medium were added. Images were taken every 10 min for $24 \mathrm{~h}$ using of a Zeiss Axiovert 200M inverted microscope with a 10x phase objective and GFP channel in an enclosed chamber under conditions of $30^{\circ} \mathrm{C}$.

Measurement of global histone $\mathbf{H 3}$ acetylation. A culture of $C$. neoformans in $2 \mathrm{ml}$ was grown in Sabouraud broth for $24 \mathrm{~h}$. Protein samples were extracted by vortexing for $4 \mathrm{~h}$ with $0.5 \mathrm{~mm}$ glass beads and yeastbuster extraction buffer (Merck, Darmstadt, Germany) at $4{ }^{\circ} \mathrm{C}$. Supernatant were collected and 100\% trichloroacetic acid was added at a 1:4 ratio. The mixtures were incubated on ice for $30 \mathrm{~min}$, and pellets were then collected by centrifugation at $13,000 \mathrm{~g}$ for 10 min at $4^{\circ} \mathrm{C}$. Pellets were washed twice with $1 \mathrm{ml}$ acetone and dissolved in $20 \mu \mathrm{l}$ water. The protein concentrations were measured by using Micro BCA ${ }^{\mathrm{TM}}$ Protein Assay Kit (Themofisher, 
Waltham, Ma). The protein samples $(3.5 \mu \mathrm{g})$ were used to detect the global histone $\mathrm{H} 3$ acetylation levels by using the EpiQuik global histone H3 acetylation assay kit (EpigenTek, Farmingdale, NY) according to manufacturer's instructions.

Stress sensitivity test. The overnight cultures were diluted in Sabouraud broth to an $\mathrm{OD}_{600}$ of 2 and further diluted 10-, 10²-, 103-, 104- , 105- fold. The dilutions $(5 \mu \mathrm{l})$ were spotted onto Sabouraud agar plates supplemented with $16 \mu \mathrm{g} / \mathrm{ml}$ fluconazole and incubated for $48 \mathrm{~h}$ at $30{ }^{\circ} \mathrm{C}$. Plates without fluconazole were also incubated for $48 \mathrm{~h}$ either 30 or $37^{\circ} \mathrm{C}$.

Growth curve. C. neoformans strains Ftc555-1, F5 and F6 were grown in Sabouraud media at $30^{\circ} \mathrm{C}$ with orbital shaker $(120 \mathrm{rpm})$ for 7 days with data measurements each 24 hours. The assay was performed in a 96-well plate and some serial dilutions were done, with a cell concentration range between $1.0 \times 10^{7}$ to $5.0 \times 10^{3} /$ well. Each condition was done in triplicate. The growth was measured by optical density at $600 \mathrm{~nm}$.

Murine Infection. Six-week-old female A/J mice were infected intranasally with $20 \mu 1$ of $1.0 \mathrm{X}$ $10^{7}$ yeast cells of each $C$. neoformans strain. Three groups of mice ( $\mathrm{n}=8$ animals per group) were infected and deaths were scored daily for 60 days. No death was observed during this time, so we decided to euthanize the animal for fungal burden assessment and cytokines levels determination.

732 A second experimental infection was performed with some modifications. Six-week-old female $733 \mathrm{~A} / \mathrm{J}$ mice were infected intranasally with $20 \mu 1$ of $1.0 \times 10^{7}$ yeast cells of each $C$. neoformans strain 734 ( $\mathrm{n}=5$ animal per group) and then euthanized after 5 days. Specific organs were removed for fungal 735 burden and cytokines level evaluation. units). After animals euthanasia, the lungs were removed, weighed and homogenized in $1 \mathrm{ml}$ of 
738 PBS. After serial dilutions, homogenates were inoculated onto Sabouraud agar plates with $10 \mathrm{U} / \mathrm{ml}$

739 of streptomycin/penicillin. The plates were incubated at room temperature, and the colonies were

740 counted after $48-72 \mathrm{~h}$.

741 Determination of cytokine levels in the organs. Spleen and lungs of each mouse were macerated

742 with protease inhibitor (complete, EDTA-free, Roche Life Science, Indiana, United States) and 743 centrifuged; supernatants of these samples were used for cytokine detection by a sandwich-ELISA

744 by using commercial kits (BD OptEIA ${ }^{\mathrm{TM}}$, BD Franklin Lakes, Nova Jersey, US) for the following

745 cytokines: IL-2 (\#555148), IL- 4 (\#555232), IL-10 (\#555252), IFN- $\gamma$ (\#551866) and TNF- $\alpha$

746 (\#555268). The protocol was followed according to the manufacturer's recommendations. The

747 reading was performed in a plate spectrophotometer at $450 \mathrm{~nm}$ and $570 \mathrm{~nm}$. 


\section{Acknowledgements}

751 We thank the Broad Institute Microbial Omics Core for generating the DNA libraries and the

752 Genomics Platform for the sequence for this study. 
756 Table 1. High and moderate impact SNPs found in passaged isolates

\begin{tabular}{|c|c|c|c|c|c|c|c|}
\hline Isolates & Chr & Position & Reference & Alternate & Gene ID & Gene function & $\begin{array}{l}\text { Effect of } \\
\text { mutation }\end{array}$ \\
\hline $\begin{array}{l}\mathrm{H} 1, \mathrm{H} 2, \\
\mathrm{H} 14\end{array}$ & 3 & 211613 & $\mathrm{~T}$ & G & CNAG_03013 & $\begin{array}{l}\text { Oligopeptide } \\
\text { transporter }\end{array}$ & M484R \\
\hline $\begin{array}{l}\mathrm{H} 1, \mathrm{H} 2, \\
\mathrm{H} 14\end{array}$ & 6 & 68953 & C & A & CNAG_02531 & $\begin{array}{l}\text { Calcium- } \\
\text { dependent } \\
\text { protein kinase }\end{array}$ & $\begin{array}{l}\text { Intron } \\
\text { variant }\end{array}$ \\
\hline A1 & 5 & 1208219 & $\mathrm{~T}$ & C & CNAG_01101 & $\begin{array}{l}\text { Hypothetical } \\
\text { protein }\end{array}$ & R478G \\
\hline $\mathrm{A} 2, \mathrm{~A} 3$ & 3 & 213165 & G & A & CNAG_03013 & $\begin{array}{l}\text { Oligopeptide } \\
\text { transporter }\end{array}$ & $\begin{array}{l}\text { Nonsense } \\
\text { mutation } \\
\text { W932* }\end{array}$ \\
\hline $\mathrm{A} 2, \mathrm{~A} 3$ & 3 & 594765 & A & G & CNAG_02858 & $\begin{array}{l}\text { Adenylsuccinate } \\
\text { synthetase }\end{array}$ & I346V \\
\hline A2 & 13 & 592173 & $C$ & $\mathrm{~T}$ & & $\begin{array}{l}\text { Intergenic } \\
\text { region }\end{array}$ & \\
\hline $\mathrm{F}^{+}$ & 3 & 213566 & G & $\mathrm{T}$ & CNAG_03013 & $\begin{array}{l}\text { Oligopeptide } \\
\text { transporter }\end{array}$ & $\begin{array}{l}\text { Splice site } \\
\text { mutation }\end{array}$ \\
\hline $\mathrm{F} 5, \mathrm{FG}^{+}$ & 1 & 1469244 & C & A & CNAG_00570 & $\begin{array}{l}\text { cAMP- } \\
\text { dependent } \\
\text { protein kinase } \\
\text { regulator }\end{array}$ & $\begin{array}{l}\text { Nonsense } \\
\text { mutation } \\
\text { G407* }\end{array}$ \\
\hline $\mathrm{FC}^{+}$ & 11 & 136455 & $\mathrm{~T}$ & G & CNAG_01506 & $\begin{array}{l}\text { Hypothetical } \\
\text { protein }\end{array}$ & $\begin{array}{l}\text { Splice site } \\
\text { mutation }\end{array}$ \\
\hline
\end{tabular}

${ }^{+}$Only high impact mutations of Ftc555-1 variants were shown in this table.

758 Table 2 High impact indels found in passaged A1-35-8 isolates

\begin{tabular}{|l|l|l|l|l|l|l|l|}
\hline Isolates & Chr & Position & Reference & Alternate & Gene ID & Gene function & $\begin{array}{l}\text { Effect of } \\
\text { mutation }\end{array}$ \\
\hline A1 & 3 & 211137 & GC & G & CNAG_03013 & $\begin{array}{l}\text { Oligopeptide } \\
\text { transporter }\end{array}$ & $\begin{array}{l}\text { Frameshift at } \\
\text { P358 }\end{array}$ \\
\hline $\begin{array}{l}\text { A4, A5, } \\
\text { A6 }\end{array}$ & 2 & 363200 & CA & C & CNAG_03622 & Cell polarity & $\begin{array}{l}\text { Frameshift at } \\
\text { N150 }\end{array}$ \\
\hline
\end{tabular}




\section{References}

1. Rajasingham R, Smith RM, Park BJ, Jarvis JN, Govender NP, Chiller TM, et al. Global burden of disease of HIV-associated cryptococcal meningitis: an updated analysis. The Lancet Infectious Diseases. 2017;0. doi:10.1016/S1473-3099(17)30243-8

2. Cross CE, Bancroft GJ. Ingestion of acapsular Cryptococcus neoformans occurs via mannose and beta-glucan receptors, resulting in cytokine production and increased phagocytosis of the encapsulated form. Infect Immun. 1995;63: 2604-2611.

3. Johnston SA, May RC. Cryptococcus interactions with macrophages: evasion and manipulation of the phagosome by a fungal pathogen. Cell Microbiol. 2013;15: 403-411. doi:10.1111/cmi.12067

4. Kozel TR. Non-encapsulated variant of Cryptococcus neoformans. II. Surface receptors for cryptococcal polysaccharide and their role in inhibition of phagocytosis by polysaccharide. Infect Immun. 1977;16: 99-106.

5. Vecchiarelli A. Immunoregulation by capsular components of Cryptococcus neoformans. Med Mycol. 2000;38: 407-417.

6. Cox GM, Mukherjee J, Cole GT, Casadevall A, Perfect JR. Urease as a Virulence Factor in Experimental Cryptococcosis. Infect Immun. 2000;68: 443-448.

7. Cox GM, McDade HC, Chen SCA, Tucker SC, Gottfredsson M, Wright LC, et al. Extracellular phospholipase activity is a virulence factor for Cryptococcus neoformans. Molecular Microbiology. 2001;39: 166-175. doi:10.1046/j.1365-2958.2001.02236.x

8. Emmons CW. ISOLATION OF CRYPTOCOCCUS NEOFORMANS FROM SOIL. J Bacteriol. 1951;62: 685690.

9. Emmons CW. Saprophytic sources of Cryptococcus neoformans associated with the pigeon (Columba livia). Am J Hyg. 1955;62: 227-232.

10. Lazera MS, Salmito Cavalcanti MA, Londero AT, Trilles L, Nishikawa MM, Wanke B. Possible primary ecological niche of Cryptococcus neoformans. Med Mycol. 2000;38: 379-383.

11. Castro e Silva DM, Santos DCS, Martins MA, Oliveira L, Szeszs MW, Melhem MSC. First isolation of Cryptococcus neoformans genotype VNI MAT-alpha from wood inside hollow trunks of Hymenaea courbaril. Med Mycol. 2016;54: 97-102. doi:10.1093/mmy/myv066

12. Casadevall A, Fu MS, Guimaraes AJ, Albuquerque P. The 'Amoeboid Predator-Fungal Animal Virulence' Hypothesis. Journal of Fungi. 2019;5: 10. doi:10.3390/jof5010010

13. Ophir T, Gutnick DL. A Role for Exopolysaccharides in the Protection of Microorganisms from Desiccation. Appl Environ Microbiol. 1994;60: 740-745.

14. Steenbergen JN, Shuman HA, Casadevall A. Cryptococcus neoformans interactions with amoebae suggest an explanation for its virulence and intracellular pathogenic strategy in macrophages. Proc Natl Acad Sci U S A. 2001;98: 15245-15250. doi:10.1073/pnas.261418798 
15. Jacobson ES, Emery HS. Catecholamine uptake, melanization, and oxygen toxicity in Cryptococcus neoformans. Journal of Bacteriology. 1991;173: 401-403. doi:10.1128/jb.173.1.401-403.1991

16. Wang $Y$, Aisen $P$, Casadevall A. Cryptococcus neoformans melanin and virulence: mechanism of action. Infection and Immunity. 1995;63: 3131-3136.

17. Rosas ÁL, Casadevall A. Melanization affects susceptibility of Cryptococcus neoformans to heat and cold. FEMS Microbiol Lett. 1997;153: 265-272. doi:10.1111/j.1574-6968.1997.tb12584.x

18. Wang Y, Casadevall A. Decreased susceptibility of melanized Cryptococcus neoformans to UV light. Appl Environ Microbiol. 1994;60: 3864-3866.

19. Fu MS, Coelho C, Leon-Rodriguez CMD, Rossi DCP, Camacho E, Jung EH, et al. Cryptococcus neoformans urease affects the outcome of intracellular pathogenesis by modulating phagolysosomal pH. PLOS Pathogens. 2018;14: e1007144. doi:10.1371/journal.ppat.1007144

20. Casadevall A, Perfect JR. Cryptococcus neoformans. American Society of Microbiology; 1998. doi:10.1128/9781555818241

21. Cirillo JD, Falkow S, Tompkins LS. Growth of Legionella pneumophila in Acanthamoeba castellanii enhances invasion. Infection and Immunity. 1994;62: 3254-3261.

22. Cirillo JD, Falkow S, Tompkins LS, Bermudez LE. Interaction of Mycobacterium avium with environmental amoebae enhances virulence. Infection and Immunity. 1997;65: 3759-3767.

23. Novohradská S, Ferling I, Hillmann F. Exploring Virulence Determinants of Filamentous Fungal Pathogens through Interactions with Soil Amoebae. Front Cell Infect Microbiol. 2017;7. doi:10.3389/fcimb.2017.00497

24. Albuquerque P, Nicola AM, Magnabosco DAG, Derengowski L da S, Crisóstomo LS, Xavier LCG, et al. A hidden battle in the dirt: Soil amoebae interactions with Paracoccidioides spp. PLOS Neglected Tropical Diseases. 2019;13: e0007742. doi:10.1371/journal.pntd.0007742

25. Davies B, Chattings LS, Edwards SW. Superoxide generation during phagocytosis by Acanthamoeba castellanii: similarities to the respiratory burst of immune phagocytes. Microbiology,. 1991;137: 705-710. doi:10.1099/00221287-137-3-705

26. Davies B, Edwards SW. Chemiluminescence and superoxide production in Acanthamoeba castellanii: free radicals generated during oxidative stress. Microbiology,. 1991;137: 1021-1027. doi:10.1099/00221287-137-5-1021

27. Allen PG, Dawidowicz EA. Phagocytosis in Acanthamoeba: I. A mannose receptor is responsible for the binding and phagocytosis of yeast. Journal of Cellular Physiology. 1990;145: 508-513. doi:10.1002/jcp.1041450317

28. Allen PG, Dawidowicz EA. Phagocytosis in Acanthamoeba: II. Soluble and insoluble mannose-rich ligands stimulate phosphoinositide metabolism. Journal of Cellular Physiology. 1990;145: 514-521. doi:10.1002/jcp.1041450318 
29. Brown RC, Bass H, Coombs JP. Carbohydrate binding proteins involved in phagocytosis by Acanthamoeba. Nature. 1975;254: 434-435. doi:10.1038/254434a0

30. Lock R, Öhman L, Dahlgren C. Phagocytic recognition mechanisms in human granulocytes and Acanthamoeba castellanii using type 1 fimbriated Escherichia coli as phagocytic prey. FEMS Microbiol Lett. 1987;44: 135-140. doi:10.1111/j.1574-6968.1987.tb02257.x

31. Gotthardt D, Warnatz HJ, Henschel O, Brückert F, Schleicher M, Soldati T. High-Resolution Dissection of Phagosome Maturation Reveals Distinct Membrane Trafficking Phases. MBoC. 2002;13: 3508-3520. doi:10.1091/mbc.e02-04-0206

32. Kwaik YA. The phagosome containing Legionella pneumophila within the protozoan Hartmannella vermiformis is surrounded by the rough endoplasmic reticulum. Appl Environ Microbiol. 1996;62: 2022-2028.

33. Bozue JA, Johnson W. Interaction of Legionella pneumophila with Acanthamoeba castellanii: Uptake by Coiling Phagocytosis and Inhibition of Phagosome-Lysosome Fusion. INFECT IMMUN. 1996;64: 6.

34. Horwitz MA. The Legionnaires' disease bacterium (Legionella pneumophila) inhibits phagosomelysosome fusion in human monocytes. Journal of Experimental Medicine. 1983;158: 2108-2126. doi:10.1084/jem.158.6.2108

35. Horwitz MA, Silverstein SC. Legionnaires' Disease Bacterium (Legionella pneumophila) Multiplies Intracellularly in Human Monocytes. J Clin Invest. 1980;66: 441-450. doi:10.1172/JCl109874

36. Rowbotham TJ. Preliminary report on the pathogenicity of Legionella pneumophila for freshwater and soil amoebae. Journal of Clinical Pathology. 1980;33: 1179-1183. doi:10.1136/jcp.33.12.1179

37. Swanson MS, Isberg RR. Association of Legionella pneumophila with the macrophage endoplasmic reticulum. Infect Immun. 1995;63: 3609-3620.

38. Arico-Muendel C, Centrella PA, Contonio BD, Morgan BA, O'Donovan G, Paradise CL, et al. Antiparasitic activities of novel, orally available fumagillin analogs. Bioorganic \& Medicinal Chemistry Letters. 2009;19: 5128-5131. doi:10.1016/j.bmcl.2009.07.029

39. Steenbergen JN, Nosanchuk JD, Malliaris SD, Casadevall A. Cryptococcus neoformans Virulence Is Enhanced after Growth in the Genetically Malleable Host Dictyostelium discoideum. Infection and Immunity. 2003;71: 4862-4872. doi:10.1128/IAI.71.9.4862-4872.2003

40. Chrisman CJ, Albuquerque P, Guimaraes AJ, Nieves E, Casadevall A. Phospholipids Trigger Cryptococcus neoformans Capsular Enlargement during Interactions with Amoebae and Macrophages. PLOS Pathogens. 2011;7: e1002047. doi:10.1371/journal.ppat.1002047

41. Castellani A. An amoeba growing in cultures of a yeast I II III IV. In: Jour Trop Med \& Hyg [Internet]. 19 Sep 1930 [cited 5 Nov 2019]. Available: https://eurekamag.com/research/030/004/030004150.php 
866

867

868

869

870

871

872

873

874

875

876

877

878

879

880

881

882

883

884

885

886

887

888

889

890

891

892

893

894

895

896

897

898

899

900

901

902

42. Castellani A. [Phagocytic and destructive action of Hartmanella castellanii (Amoeba castellanii) on pathogenic encapsulated yeast-like fungi Torulopsis neoformans (Cryptococcus neoformans)]. Ann Inst Pasteur (Paris). 1955;89: 1-7.

43. Ruiz A, Neilson JB, Bulmer GS. Control of Cryptococcus neoformans in nature by biotic factors. Sabouraudia. 1982;20: 21-29.

44. Garcia-Solache MA, Izquierdo-Garcia D, Smith C, Bergman A, Casadevall A. Fungal virulence in a lepidopteran model is an emergent property with deterministic features. mBio. 2013;4: e0010000113. doi:10.1128/mBio.00100-13

45. Fu MS, Casadevall A. Divalent Metal Cations Potentiate the Predatory Capacity of Amoeba for Cryptococcus neoformans. Appl Environ Microbiol. 2018;84. doi:10.1128/AEM.01717-17

46. Chrisman CJ, Alvarez M, Casadevall A. Phagocytosis of Cryptococcus neoformans by, and Nonlytic Exocytosis from, Acanthamoeba castellanii. Appl Environ Microbiol. 2010;76: 6056-6062. doi:10.1128/AEM.00812-10

47. Watkins RA, Andrews A, Wynn C, Barisch C, King JS, Johnston SA. Cryptococcus neoformans Escape From Dictyostelium Amoeba by Both WASH-Mediated Constitutive Exocytosis and Vomocytosis. Front Cell Infect Microbiol. 2018;8. doi:10.3389/fcimb.2018.00108

48. Derengowski L da S, Paes HC, Albuquerque P, Tavares AHFP, Fernandes L, Silva-Pereira I, et al. The Transcriptional Response of Cryptococcus neoformans to Ingestion by Acanthamoeba castellanii and Macrophages Provides Insights into the Evolutionary Adaptation to the Mammalian Host. Eukaryotic Cell. 2013;12: 761-774. doi:10.1128/EC.00073-13

49. Okagaki LH, Strain AK, Nielsen JN, Charlier C, Baltes NJ, Chrétien F, et al. Cryptococcal Cell Morphology Affects Host Cell Interactions and Pathogenicity. PLOS Pathogens. 2010;6: e1000953. doi:10.1371/journal.ppat.1000953

50. Zaragoza O, García-Rodas R, Nosanchuk JD, Cuenca-Estrella M, Rodríguez-Tudela JL, Casadevall A. Fungal Cell Gigantism during Mammalian Infection. PLOS Pathogens. 2010;6: e1000945. doi:10.1371/journal.ppat.1000945

51. Okagaki LH, Nielsen K. Titan Cells Confer Protection from Phagocytosis in Cryptococcus neoformans Infections. Eukaryot Cell. 2012;11: 820-826. doi:10.1128/EC.00121-12

52. Magditch DA, Liu T-B, Xue C, Idnurm A. DNA Mutations Mediate Microevolution between HostAdapted Forms of the Pathogenic Fungus Cryptococcus neoformans. PLOS Pathogens. 2012;8: e1002936. doi:10.1371/journal.ppat.1002936

53. Homer CM, Summers DK, Goranov Al, Clarke SC, Wiesner DL, Diedrich JK, et al. Intracellular Action of a Secreted Peptide Required for Fungal Virulence. Cell Host \& Microbe. 2016;19: 849-864. doi:10.1016/j.chom.2016.05.001

54. Gerstein AC, Fu MS, Mukaremera L, Li Z, Ormerod KL, Fraser JA, et al. Polyploid Titan Cells Produce Haploid and Aneuploid Progeny To Promote Stress Adaptation. mBio. 2015;6.

doi:10.1128/mBio.01340-15 
903

904

905

906

907

908

909

910

911

912

913

914

915

916

917

918

919

920

921

922

923

924

925

926

927

928

929

930

931

932

933

934

935

936

937

938

939

55. Sionov E, Lee H, Chang YC, Kwon-Chung KJ. Cryptococcus neoformans Overcomes Stress of Azole Drugs by Formation of Disomy in Specific Multiple Chromosomes. PLOS Pathogens. 2010;6: e1000848. doi:10.1371/journal.ppat.1000848

56. Anderson $\mathrm{CA}$, Roberts $\mathrm{S}$, Zhang $\mathrm{H}$, Kelly $\mathrm{CM}$, Kendall $\mathrm{A}$, Lee $\mathrm{C}$, et al. Ploidy variation in multinucleate cells changes under stress. MBoC. 2015;26: 1129-1140. doi:10.1091/mbc.E14-09-1375

57. O'Meara TR, Hay C, Price MS, Giles S, Alspaugh JA. Cryptococcus neoformans Histone Acetyltransferase Gcn5 Regulates Fungal Adaptation to the Host. Eukaryot Cell. 2010;9: 11931202. doi:10.1128/EC.00098-10

58. D'Souza CA, Alspaugh JA, Yue C, Harashima T, Cox GM, Perfect JR, et al. Cyclic AMP-Dependent Protein Kinase Controls Virulence of the Fungal Pathogen Cryptococcus neoformans. Molecular and Cellular Biology. 2001;21: 3179-3191. doi:10.1128/MCB.21.9.3179-3191.2001

59. Beenhouwer DO, Shapiro S, Feldmesser M, Casadevall A, Scharff MD. Both Th1 and Th2 Cytokines Affect the Ability of Monoclonal Antibodies To Protect Mice against Cryptococcus neoformans. Kozel TR, editor. Infect Immun. 2001;69: 6445-6455. doi:10.1128/IAI.69.10.6445-6455.2001

60. Van Waeyenberghe L, Baré J, Pasmans F, Claeys M, Bert W, Haesebrouck F, et al. Interaction of Aspergillus fumigatus conidia with Acanthamoeba castellanii parallels macrophage-fungus interactions. Environ Microbiol Rep. 2013;5: 819-824. doi:10.1111/1758-2229.12082

61. Neilson JB, Ivey MH, Bulmer GS. Cryptococcus neoformans: pseudohyphal forms surviving culture with Acanthamoeba polyphaga. Infect Immun. 1978;20: 262-266.

62. Radosa S, Ferling I, Sprague JL, Westermann M, Hillmann F. The different morphologies of yeast and filamentous fungi trigger distinct killing and feeding mechanisms in a fungivorous amoeba. Environ Microbiol. 2019;21: 1809-1820. doi:10.1111/1462-2920.14588

63. Billmyre RB, Clancey SA, Heitman J. Natural mismatch repair mutations mediate phenotypic diversity and drug resistance in Cryptococcus deuterogattii. eLife. 6. doi:10.7554/eLife.28802

64. Desjardins CA, Giamberardino C, Sykes SM, Yu C-H, Tenor JL, Chen Y, et al. Population genomics and the evolution of virulence in the fungal pathogen Cryptococcus neoformans. Genome Res. 2017;27: 1207-1219. doi:10.1101/gr.218727.116

65. Ormerod KL, Morrow CA, Chow EWL, Lee IR, Arras SDM, Schirra HJ, et al. Comparative Genomics of Serial Isolates of Cryptococcus neoformans Reveals Gene Associated With Carbon Utilization and Virulence. G3: Genes, Genomes, Genetics. 2013;3: 675-686. doi:10.1534/g3.113.005660

66. Hommel B, Mukaremera L, Cordero RJB, Coelho C, Desjardins CA, Sturny-Leclère A, et al. Titan cells formation in Cryptococcus neoformans is finely tuned by environmental conditions and modulated by positive and negative genetic regulators. PLOS Pathogens. 2018;14: e1006982. doi:10.1371/journal.ppat.1006982

67. Torres EM, Sokolsky T, Tucker CM, Chan LY, Boselli M, Dunham MJ, et al. Effects of aneuploidy on cellular physiology and cell division in haploid yeast. Science. 2007;317: 916-924.

doi:10.1126/science.1142210 
68. Rancati G, Pavelka N, Fleharty B, Noll A, Trimble R, Walton K, et al. Aneuploidy Underlies Rapid Adaptive Evolution of Yeast Cells Deprived of a Conserved Cytokinesis Motor. Cell. 2008;135: 879893. doi:10.1016/j.cell.2008.09.039

69. Pavelka N, Rancati G, Zhu J, Bradford WD, Saraf A, Florens L, et al. Aneuploidy confers quantitative proteome changes and phenotypic variation in budding yeast. Nature. 2010;468: 321-325. doi:10.1038/nature09529

70. Selmecki A, Forche A, Berman J. Aneuploidy and Isochromosome Formation in Drug-Resistant Candida albicans. Science. 2006;313: 367-370. doi:10.1126/science.1128242

71. Selmecki A, Gerami-Nejad M, Paulson C, Forche A, Berman J. An isochromosome confers drug resistance in vivo by amplification of two genes, ERG11 and TAC1. Molecular Microbiology. 2008;68: 624-641. doi:10.1111/j.1365-2958.2008.06176.x

72. Sunshine AB, Payen C, Ong GT, Liachko I, Tan KM, Dunham MJ. The Fitness Consequences of Aneuploidy Are Driven by Condition-Dependent Gene Effects. PLOS Biology. 2015;13: e1002155. doi:10.1371/journal.pbio.1002155

73. Lin J, Idnurm A, Lin X. Morphology and its underlying genetic regulation impact the interaction between Cryptococcus neoformans and its hosts. Med Mycol. 2015;53: 493-504. doi:10.1093/mmy/myv012

74. Walton FJ, Heitman J, Idnurm A. Conserved Elements of the RAM Signaling Pathway Establish Cell Polarity in the Basidiomycete Cryptococcus neoformans in a Divergent Fashion from Other Fungi. MBoC. 2006;17: 3768-3780. doi:10.1091/mbc.e06-02-0125

75. Thomson DD, Berman J, Brand AC. High frame-rate resolution of cell division during Candida albicans filamentation. Fungal Genet Biol. 2016;88: 54-58. doi:10.1016/j.fgb.2016.02.001

76. Gonçalves D de S, Ferreira M da S, Gomes KX, Noval CRL, Liedke SC, Costa GCV da, et al. Unravelling the interactions of the environmental host Acanthamoeba castellanii with fungi through the recognition by mannose-binding proteins. Cellular Microbiology. 2019;21: e13066. doi:10.1111/cmi.13066

77. Casadevall A. The Pathogenic Potential of a Microbe. mSphere. 2017;2. doi:10.1128/mSphere.00015-17

78. Chen Y, Farrer RA, Giamberardino C, Sakthikumar S, Jones A, Yang T, et al. Microevolution of Serial Clinical Isolates of Cryptococcus neoformans var. grubii and C. gattii. mBio. 2017;8. doi:10.1128/mBio.00166-17

79. Blasi E, Brozzetti A, Francisci D, Neglia R, Cardinali G, Bistoni F, et al. Evidence of Microevolution in a Clinical Case of Recurrent Cryptococcus neoformans Meningoencephalitis. Eur J Clin Microbiol Infect Dis. 2001;20: 535-543. doi:10.1007/s100960100549

80. Sullivan D, Haynes K, Moran G, Shanley D, Coleman D. Persistence, replacement, and microevolution of Cryptococcus neoformans strains in recurrent meningitis in AIDS patients. Journal of Clinical Microbiology. 1996;34: 1739-1744. 
81. Chang YC, Lamichhane AK, Kwon-Chung KJ. Cryptococcus neoformans, Unlike Candida albicans, Forms Aneuploid Clones Directly from Uninucleated Cells under Fluconazole Stress. mBio. 2018;9. doi:10.1128/mBio.01290-18

82. Roberts GD, Horstmeier CD, Land GA, Foxworth JH. Rapid urea broth test for yeasts. J Clin Microbiol. 1978;7: 584-588.

83. Kwon-Chung KJ, Wickes BL, Booth JL, Vishniac HS, Bennett JE. Urease inhibition by EDTA in the two varieties of Cryptococcus neoformans. Infect Immun. 1987;55: 1751-1754.

84. Velegraki A, Kambouris M, Kostourou A, Chalevelakis G, Legakis NJ. Rapid extraction of fungal DNA from clinical samples for PCR amplification. Medical Mycology. 1999; 5.

85. Janbon G, Ormerod KL, Paulet D, Byrnes EJ, Yadav V, Chatterjee G, et al. Analysis of the genome and transcriptome of Cryptococcus neoformans var. grubii reveals complex RNA expression and microevolution leading to virulence attenuation. PLoS Genet. 2014;10: e1004261. doi:10.1371/journal.pgen.1004261

86. Li H. Aligning sequence reads, clone sequences and assembly contigs with BWA-MEM. arXiv:13033997 [q-bio]. 2013 [cited 22 May 2015]. Available: http://arxiv.org/abs/1303.3997

87. McKenna A, Hanna M, Banks E, Sivachenko A, Cibulskis K, Kernytsky A, et al. The Genome Analysis Toolkit: a MapReduce framework for analyzing next-generation DNA sequencing data. Genome Res. 2010;20: 1297-303. doi:10.1101/gr.107524.110

88. Cingolani $\mathrm{P}$, Platts $\mathrm{A}$, Wang LL, Coon $\mathrm{M}$, Nguyen $\mathrm{T}$, Wang $\mathrm{L}$, et al. A program for annotating and predicting the effects of single nucleotide polymorphisms, SnpEff. Fly (Austin). 2012;6: 80-92. doi:10.4161/fly.19695 


\section{Figure legends}

1000

1001

1002

1003

1004

1005

1006

1007

1008

1009

1010

1011

1012

1013

1014

1015

1016

1017

1018

1019

1020

1021

Fig 1. C. neoformans colonies exhibit various cellular and colony morphologies after co-

incubated with amoebae in Sabouraud agar. (A) Small colonies of H99 (red arrow) surviving in a mat of amoeba that appears a hazy cloudy area (denoted by dashed line). Typical C. neoformans colonies $(\mathrm{CN})$ are visible on the right bottom of the image. (B) Cells in the survival colony exhibit hyphal or pseudohyphal morphology (100× magnification). (C) Both pseudohyphae and yeast cells were identified on a wet mount of samples taken from the survival colony (400x). (D) Schematic representation of individual hyphae isolation. i. Survival colonies were picked using pipette tips and transferred to PBS in $3 \mathrm{~mm}$ culture dish. ii. Total 20 individual hyphae from 2-4 colonies were selected under microscope and transferred to fresh Sabouraud agar. Plates were incubated at $30^{\circ} \mathrm{C}$ to generate colonies. iii. Six colonies were then selected for further phenotypic characterization. iv. Control colonies were also picked from the same plate of hyphal isolates but without interacting with amoeba. (E) Single pseudohyphal cell has been isolated from the survival colonies and transferred onto a fresh amoebae-free solid medium where form new colonies. (F) Microcolony with mostly yeast cells has been formed from a single pseudohyphal cell in 24 h. (G) Colony developed a serrated appearance after 2 days. (H) Yeast cells were identified on a wet mount of samples taken from the serrated colony. (I-L) Images showed another example of single pseudohyphal cell isolation. Smooth colony was formed from this particular pseudohyphal cell. (M-N) Same experiment was performed on environmental strains A1-35-8 and Ftc-555-1. Various cellular and colony morphologies have been identified among isolates A1-A6 and F1-F6 in the background of A1-35-8 and Ftc555-1 respectively. Colonies grew up from individual hyphae which was isolated from the same survival colony were grouped in red boxes. 
1022 Fig 2. Isolates in H99 background derived from exposure to amoeba demonstrated increased 1023 resistance to amoebal killing by rapid pseudohyphal formation. (A) Scheme of amoebae killing 1024 assay. C. neoformans was streaked in a cross while $A$. castellanii was dropped at the intersection 1025 of the cross on Sabouraud agar. The data shown are the average of the distance between 1026 boundary and center of clear predator zone in four indicated directions (a-b), with the area being 1027 the predation zone. (B) All of the isolates that had prior exposure to amoeba had smaller clear 1028 zone than their ancestor and controls, consistent with enhanced resistance. A, ancestor; C1-3, 1029 controls; $\mathrm{H}$, isolates derived from H99 after exposure to amoeba. Data are means from three 1030 biological replicates and error bars are SD. (C) Samples were taken from the peripheral areas of 1031 the predator zone after one-week co-incubation with amoebae and visualized under microscope. 1032 All of the isolates showed pseudohyphal formation, but ancestor and controls did not. (D) 1033 Sample were taken from the end of the cross where $C$. neoformans have not contacted with $A$. 1034 castellanii yet. All of the isolates manifested yeast cell morphology.

1035 Fig 3. Some of the isolated recovered from the environmental strains A1-35-8 and Ftc-555-1 1036 exhibited increased resistance to $A$. castellanii. (A) No clear predator zone of clearance was 1037 apparent with isolates A4-A6, while larger predator zones were apparent for isolates A1-A3 1038 when comparing to their ancestor. A, ancestor; C1-3, controls; A1-6, isolates derived from A1$1039 \quad 35-8$ after exposure to amoeba (B) Isolates F3- F5 showed smaller predator zone than their 1040 ancestor. Data are means from three biological replicates and error bars are SD. A, ancestor; C11041 3, controls; F1-6, isolates derived from Ftc555-1 after exposure to amoeba (C) Ftc-555-1 1042 samples were collected from predator zone after one-week co-incubation. Isolates F3-F6 formed 1043 larger cell size than their ancestor and controls. (D) The cell size of isolates F3-F6 from the end 
1044 of the cross is slightly larger than the one of ancestor and controls, but they are not as large as the 1045 cells taken from predator zone.

1046 Fig 4. Capsule thickness for cells of parent strain and amoeba-selected strains. (A) H99 isolates 1047 (B) A1-35-8 isolates and (C) Ftc555-1 isolates have been cultured in minimal medium at $30{ }^{\circ} \mathrm{C}$ 1048 for three days. Capsule was visualized by counterstaining with India ink. A, ancestor; C1-3, 1049 controls. * $P<0.1 * * P<0.01 * * * * P<0.0001$ by One-way ANOVA, followed by Tukey's 1050 multiple-comparison test.

1051 Fig 5. Cellular dimensions for cells of parent strain and amoeba-selected strains. (A) H99 isolates 1052 (B) A1-35-8 isolates and (C) Ftc555-1 isolates have been cultured in minimal medium for three 1053 days. A, ancestor; C1-3, controls. (D-E) Ftc555-1 isolates have also been cultured in macrophage 1054 medium and with BMDM at $37{ }^{\circ} \mathrm{C} 9.5 \% \mathrm{CO}_{2}$ for $24 \mathrm{~h}$. Extracellular cryptococcal cells were 1055 collected from the culture supernatant while intracellular cells were retrieved from lysing the 1056 BMDM. A, ancestor; C1-3, controls. **** $P<0.0001$ by One-way ANOVA, followed by 1057 Tukey's multiple-comparison test.

1058 Fig 6. Urease activity for cells of parent strain and amoeba-selected strains. The urease activity 1059 of cryptococcal cells were detected by using rapid urea broth (RUH) method. Amoeba-passaged 1060 isolates with numbers preceded by the letters $\mathrm{H}, \mathrm{A}$, and $\mathrm{F}$ to indicate their origin from strains 1061 H99, A1-35-8 and Ftc555-1, respectively. A, ancestor; C1-3, controls. The assay was performed 1062 in triplicate for each time point. Error bars represent SD. $* P<0.1 * * P<0.01 * * * P<0.001$ by 1063 unpaired t-test.

1064 Fig 7. Melanization formation for cells of parent strain and amoeba-selected strains.

1065 Melanization was analyzed by spotting the $10^{6}$ cryptococcal cells on minimal medium agar with 
1066

1067

1068

1069

1070

1071

1072

1073

1074

1075

1076

1077

1078

1079

1080

1081

1082

1083

1084

1085

1086

1087

1088

L-DOPA for $24 \mathrm{~h}$. The pigmentation of colony was measured through grayscale pixel quantification by the software ImageJ. Relative blackness was calculated as a ratio of grayscale quantification between isolates, their ancestor (A) and control (C1-3). Error bars represent SD. * $P<0.1 * * P<0.01 * * * * P<0.0001$ by unpaired t-test.

Fig 8. The growth of parents and isolates under stress conditions. Cells were 10-fold serially diluted and spotted onto YPD medium with or without fluconazole $(16 \mu \mathrm{g} / \mathrm{ml})$, and grown for

two days at $30^{\circ} \mathrm{C}$ or $40^{\circ} \mathrm{C}$. Amoeba-passaged isolates with numbers preceded by the letters $\mathrm{H}, \mathrm{A}$, and $\mathrm{F}$ to indicate their origin from strains H99, A1-35-8 and Ftc555-1, respectively. A, ancestor; C1-3, controls.

Fig 9. Aneuploidy plays a role in pseudohyphal formation. (A) Chromosomal copy numbers of H99 isolates were determined based on depth of sequence coverage normalized by the average genome-wide sequence depth (B) Relative chromosome copy number of isolate H17 was obtained by qPCR. H17 have duplication of chromosome 8 (C) chromosome duplication in H17 is eliminated by passaging H17 in fresh Sabouraud medium for 30 days. (D) H17 euploid (H17 ${ }^{\mathrm{Eu}}$ ) strain did not form pseudohyphae as rapid as H17 aneuploid strain. (E) H17 ${ }^{\mathrm{Eu}}$ euploid strain has larger predator zone than H17 ${ }^{\text {Aneu }}$. Data represent the mean of three biological replicates per biological sample and error bars are SD. (F) $\mathrm{H} 17^{\mathrm{Eu}}$ strain has lower urease activity then $\mathrm{H} 17^{\text {Aneu }}$ and comparable urease activity as H99 at early time point ( $\left.1 \mathrm{~h}\right)$ Data represent the mean of two biological replicates per biological sample and error bars are SD. (G) H17 ${ }^{\mathrm{Eu}}$ has smaller capsule size than H17 Aneu , but similar capsule size with H99.

Fig 10. Time-lapse imaging showing nuclear division of pseudohyphae. The images of pseudohyphae of amoeba-passaged H99 GFP-H2B strain were taken by phase-contrast and fluorescence microscopy. Bud (red arrow) were forming between 0-220 min. The nucleus 
1089 migrates into the daughter cells at $240 \mathrm{~min}$, and separated at $300 \mathrm{~min}$. Nuclear division was

1090 completed at $400 \mathrm{~min}$. However, the nucleus from mother cells re-entered into the daughter cells

1091 at $500 \mathrm{~min}$ and underwent fusion at $580 \mathrm{~min}$.

1092 Fig 11. (A-C) The survival of parents and isolates in culture with BMDM. The survival of (A)

1093 H99, (B) A1-35-8 and (C) Ftc555-1 isolates was determined by colony form unit (CFU) after 0

1094 and $24 \mathrm{~h}$ phagocytosis. The percentage of survival was calculated by normalizing the CFU value

1095 of $24 \mathrm{~h}$ infection to time zero. A, ancestor; C1-3, controls. Data represent the mean of three

1096 biological replicates and error bars are SD. (D) BMDM were infected with Ftc555-1 isolates for

109748 h. LDH release from damaged BMDM into culture supernatant was assayed. $* * * P<0.001$

1098 by One-way ANOVA, followed by Tukey's multiple-comparison test.

1099 Fig 12. Fungal burden and cytokine production in lung after infection with Ftc555-1 as well as its

1100 isolates F5 and F6. After 60 days of infection, mice were sacrificed and (A) fungal burden in the

1101 lung were determined by CFU counting. The level of cytokines (B) IL4 (C) IL10 (D) IFN- $\gamma$ (E)

1102 TNF- $\alpha$ in the lung were measured by ELISA. At 5-day post-infection, (F) fungal burden, and the

1103 amount of cytokines (G) IL4 (H) IL10 (I) IFN- $\gamma$ (J) TNF- $\alpha$ in the lung were also measured. All

1104 data represent the mean of eight mice per group and errors bars are SD. $* P<0.1 * * P<0.01$. For

1105 determination of cytokine levels, one-way ANOVA with Kruskal-Wallis nonparametric test was

1106 used and followed by Bonferroni's multiple-comparison test. The t-test was used to compare the

1107 number of colony forming units (CFU) for different groups. 


\section{Supporting information}

1110 S1 Table. High impact indels found in passaged Ftc555-1 isolates

1111 S1 Fig. Relative chromosome copy number of isolate H14 was obtained by qPCR.

1112 S2 Fig. Amoebae killing assay on C. neoformans deletion mutants. Mutants showed comparable

1113 predator zone with their parental strains.

1114 S3 Fig. Global histone H3 acetylation levels in parents and isolates. Data are means of two 1115 independent experiments with standard deviations.

1116 S4 Fig. The growth curves of Ftc555-1, F5 and F6 strains with high $\left(1.0 \times 10^{7}\right)$ and low $\left(5.0 \times 10^{3}\right)$ 1117 inoculum concentration in Sabouraud medium for seven days.

1118 S5 Fig. Cytokine production in spleen of mice after infection with Ftc555-1 as well as its isolates

1119 F5 and F6. After 60 and 5 days of infection, mice were sacrificed and the level of cytokines (A, E)

1120 IL4 (B, F) IL10 (D) IFN- $\gamma(\mathrm{D}, \mathrm{G})$ TNF- $\alpha$ in the lung were measured by ELISA. All data represent

1121 the mean of eight mice per group and errors bars are SD. For determination of cytokine levels, 1122 one-way ANOVA with Kruskal-Wallis nonparametric test was used and followed by Bonferroni's 1123 multiple-comparison test. The t-test was used to compare the number of colony forming units 1124 (CFU) for different groups.

1125 S6 Fig. Virulence of parents and variant isolates in the G. mellonella larvae infection model. The 1126 Kaplan-Meier plots shows the survival of G. mellonella after injection of cryptococcal cells $\left(10^{3}\right.$

1127 cells/larva).

1128 S7 Fig. Summary of phenotypic changes occurred in amoeba-passaged isolates

1129 S1 Movie. Time-lapse imaging showing nuclear division of pseudohyphae. 
Figure 2

A
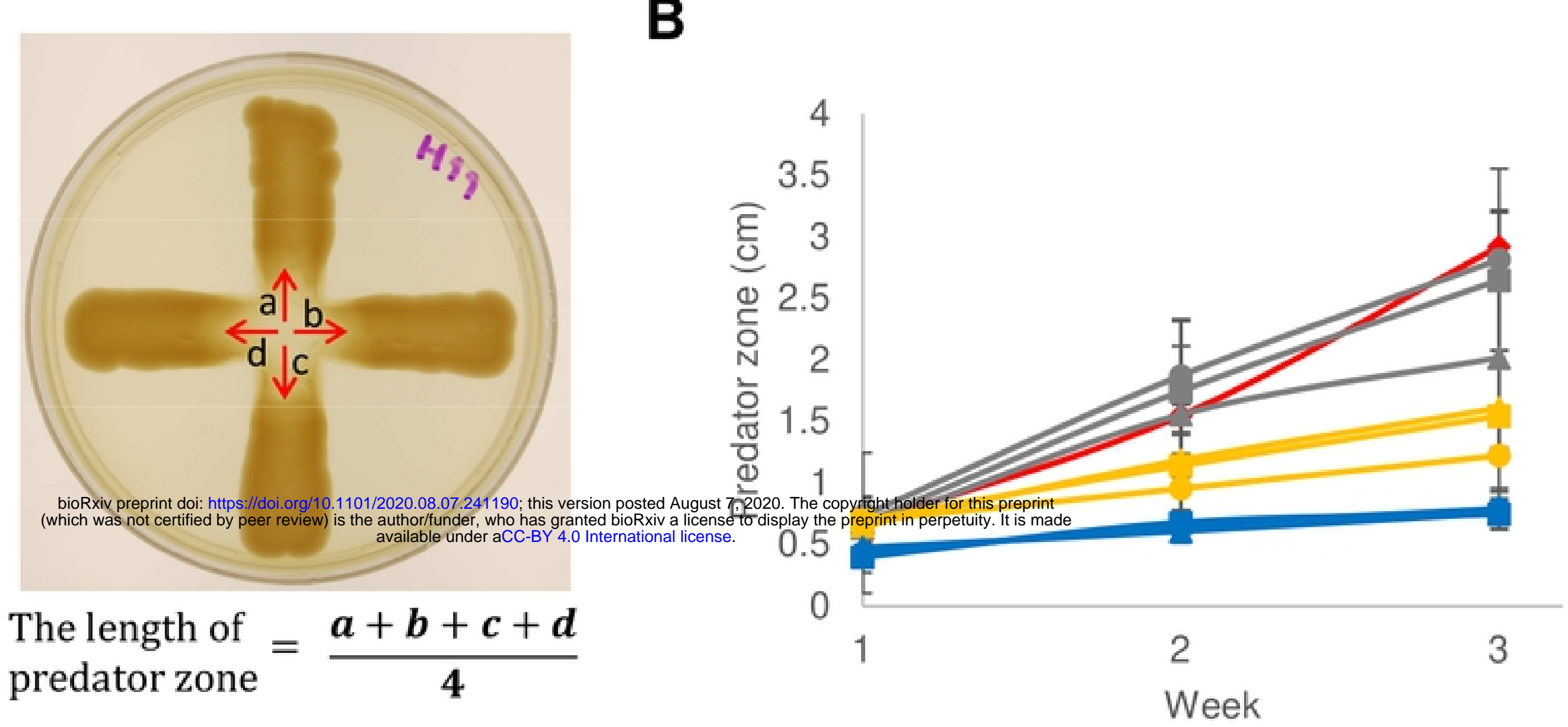

$\rightarrow$ - A

bioRxiv preprint doi: https://doi.org/10.1101/2020.08.07.241190; this version posted August 7 (which was not certified by peer review) is the authorffunder, who has granted bioRxiv a license

$\begin{aligned} & \text { The length of } \\ & \text { predator zone }\end{aligned}=\frac{\boldsymbol{a}+\boldsymbol{b}+\boldsymbol{c}+\boldsymbol{d}}{\mathbf{4}}$

Week

D

In the predator zone

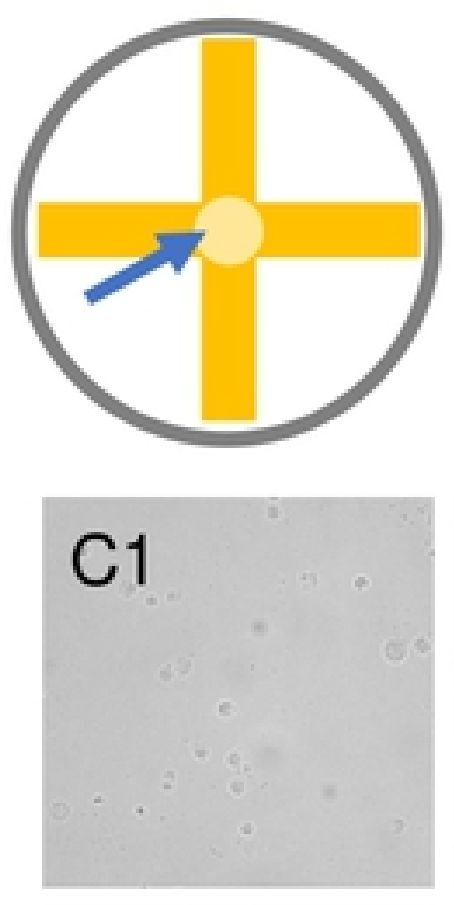

$\mathrm{H}$

$\mathrm{H} 13$
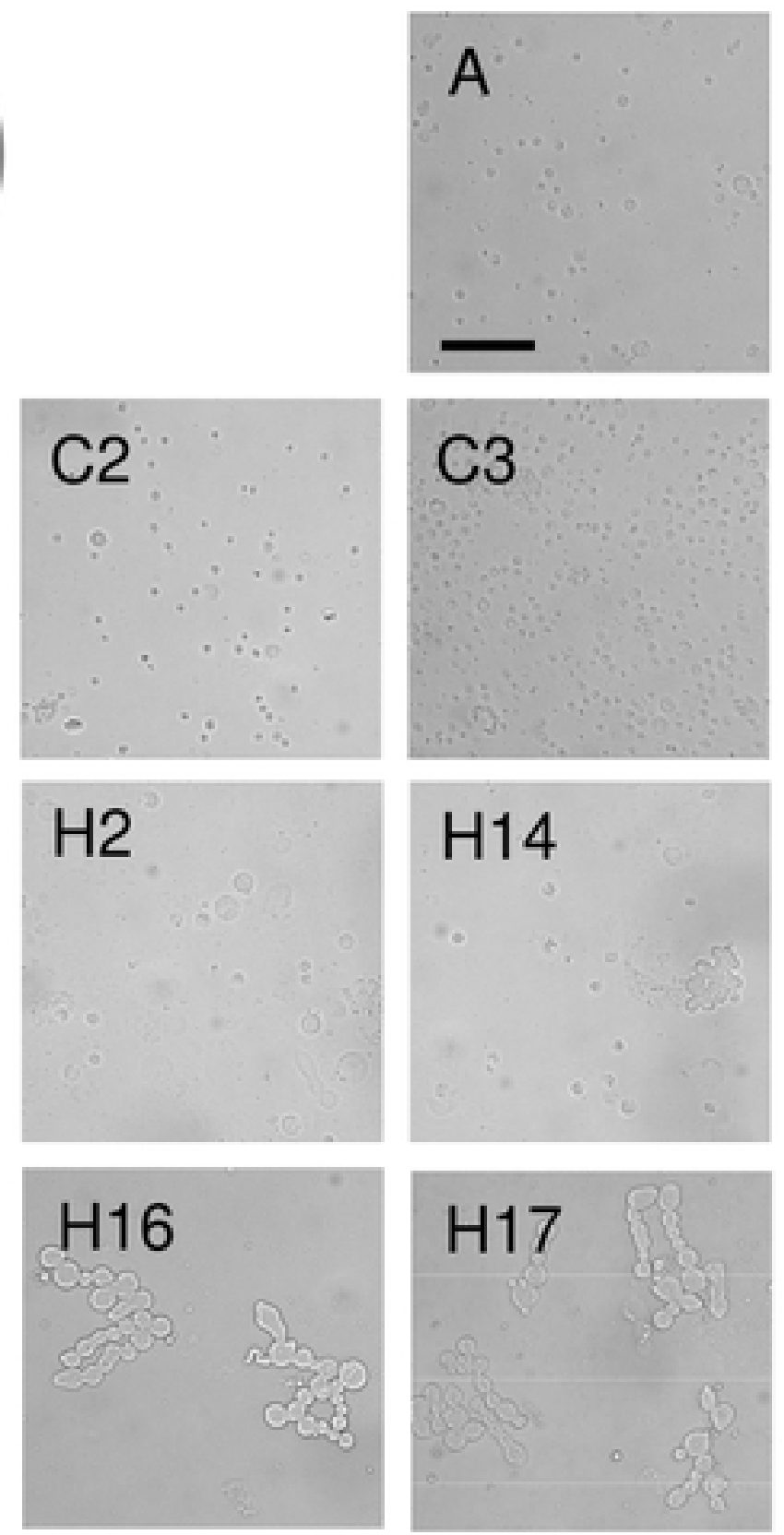

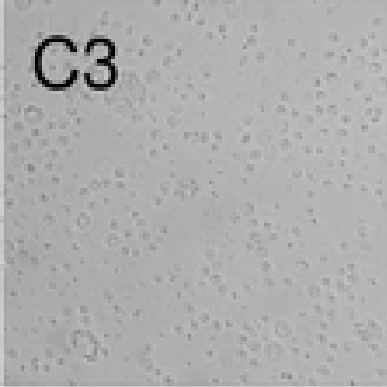

\section{$\mathrm{H} 14$}

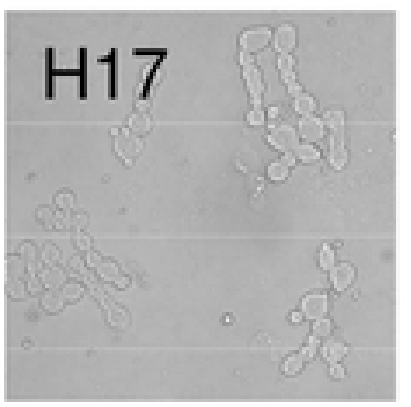

At the end of $\mathrm{CN}$ cross
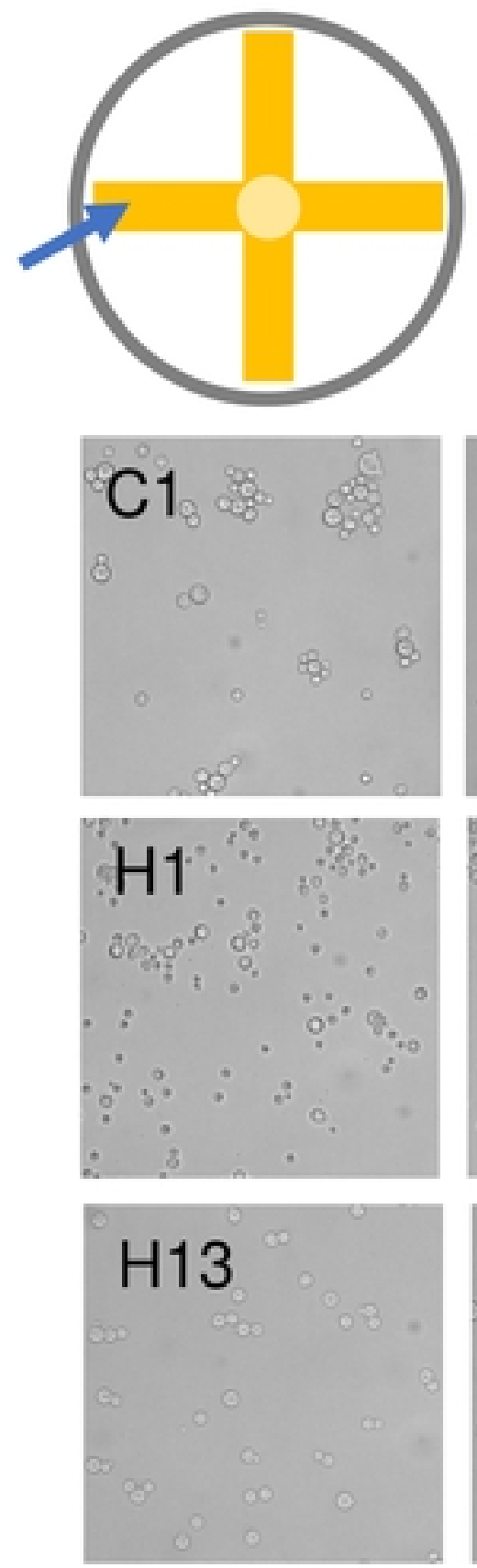

$\mathrm{H} 2$

$\mathrm{H} 14$

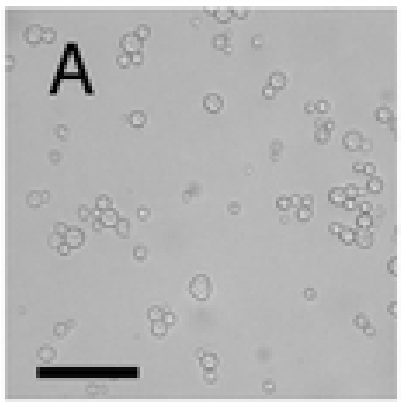

C3
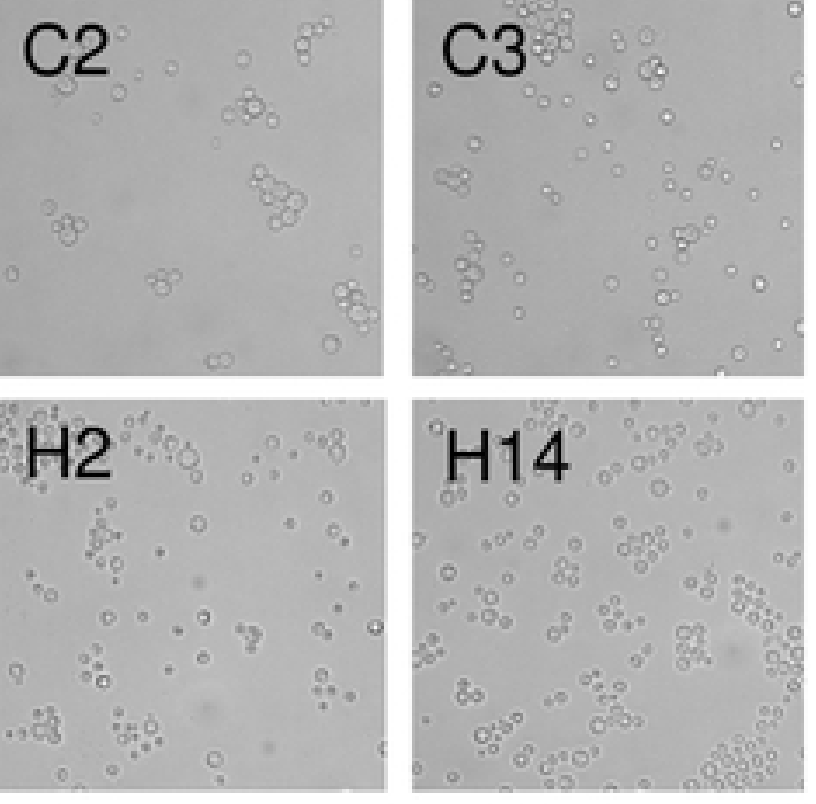

H16

Figure 2 
A

A1-35-8

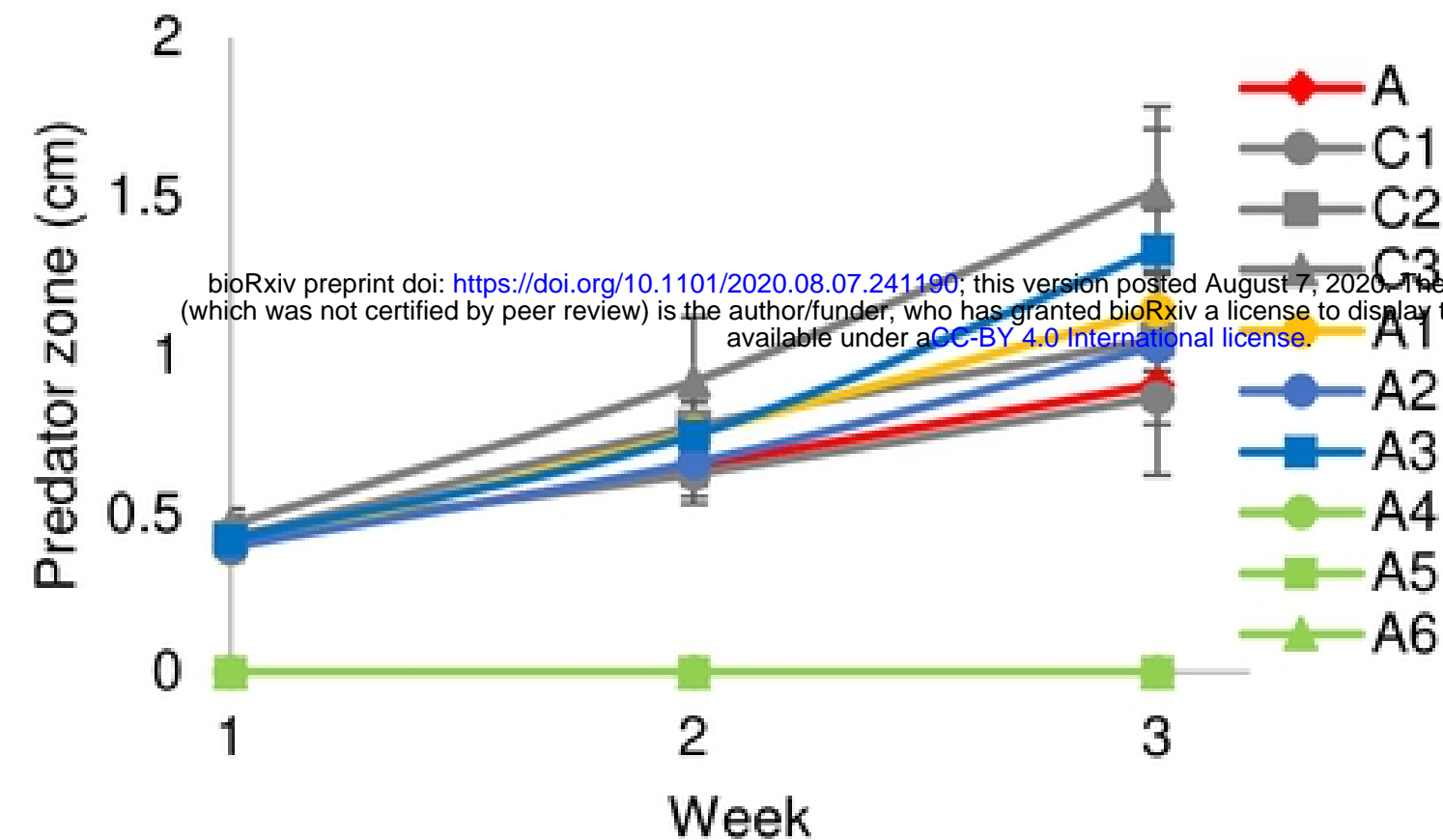

C

In the predator zone
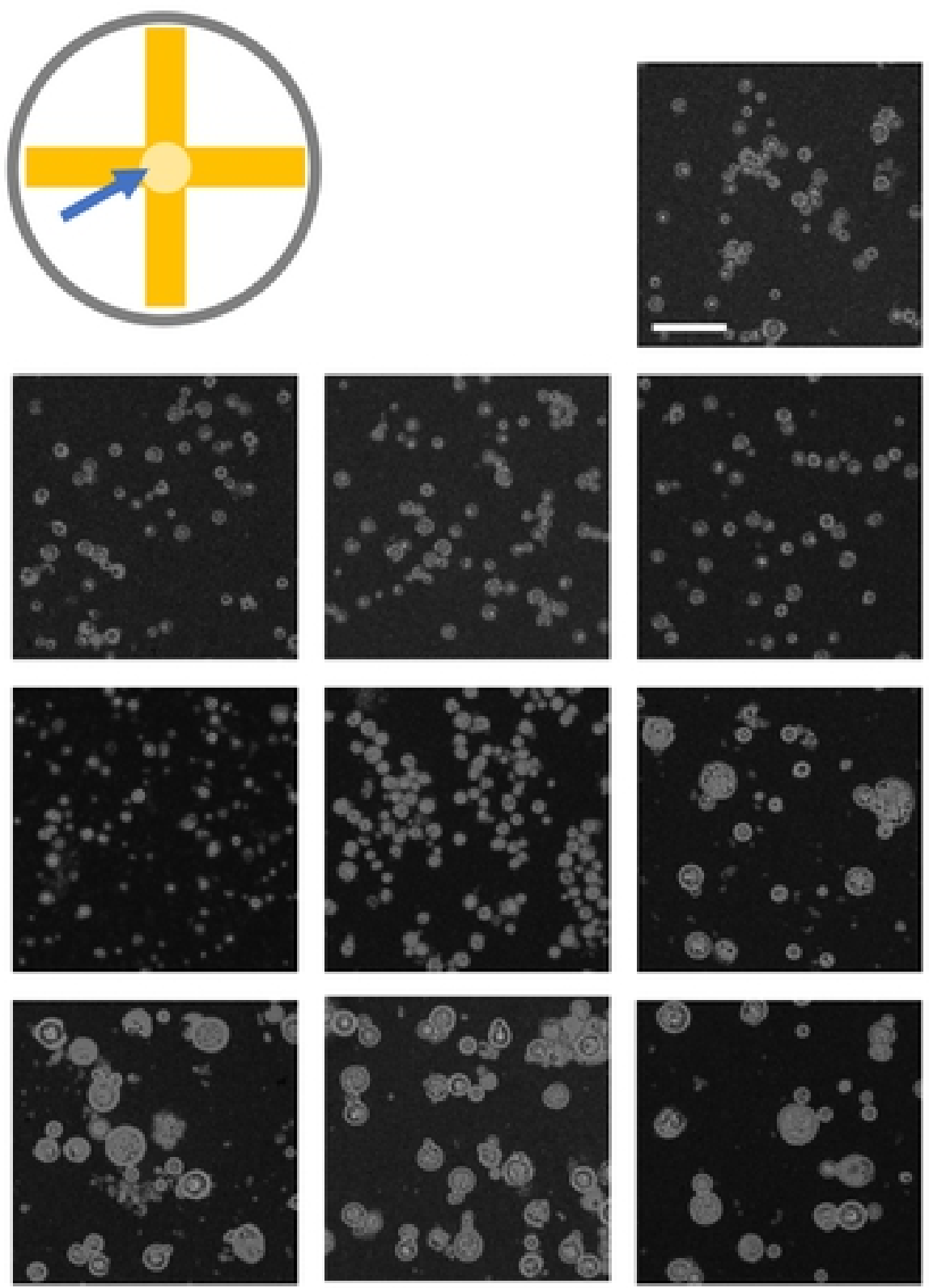

B

Ftc555-1

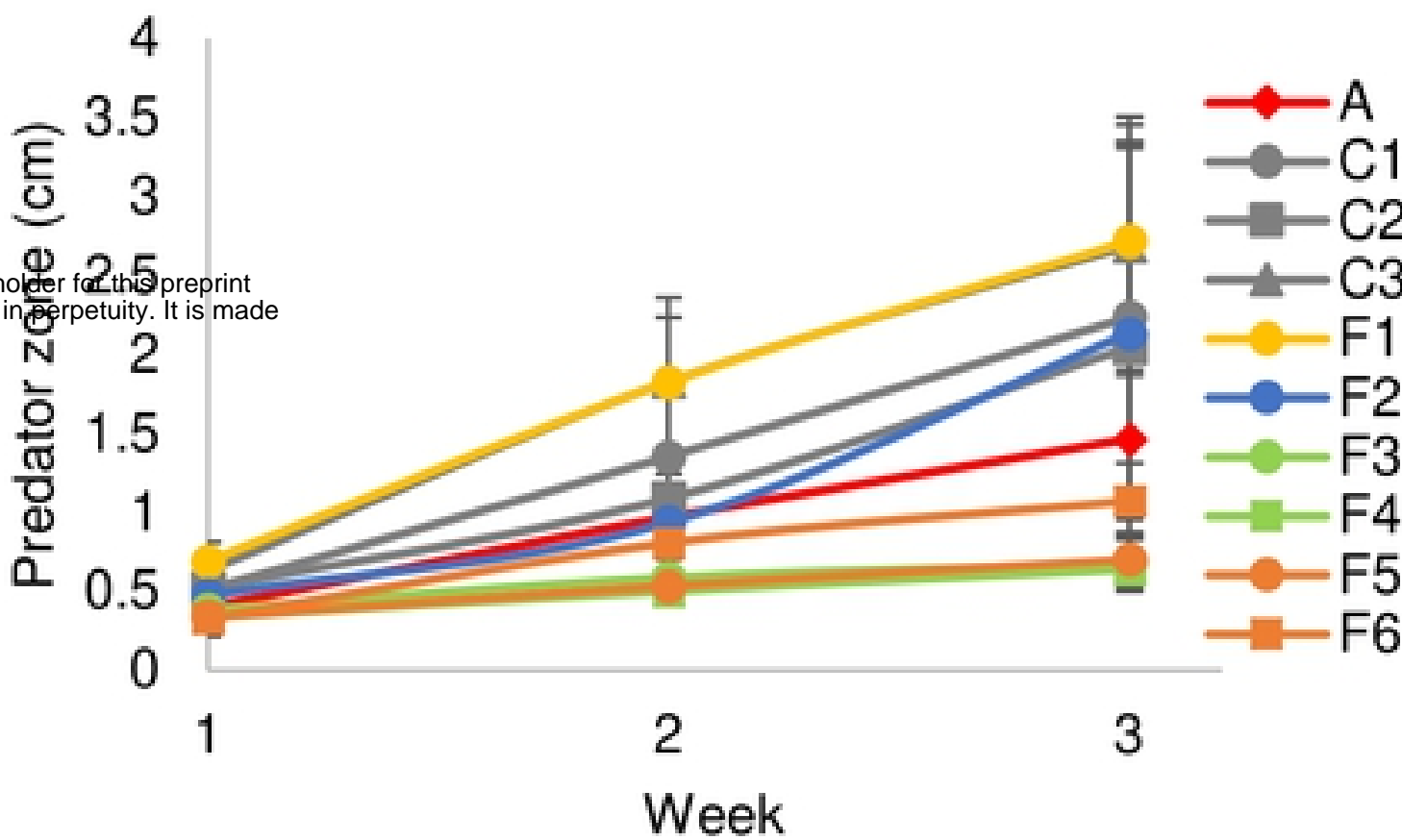

D

At the end of $\mathrm{CN}$ cross
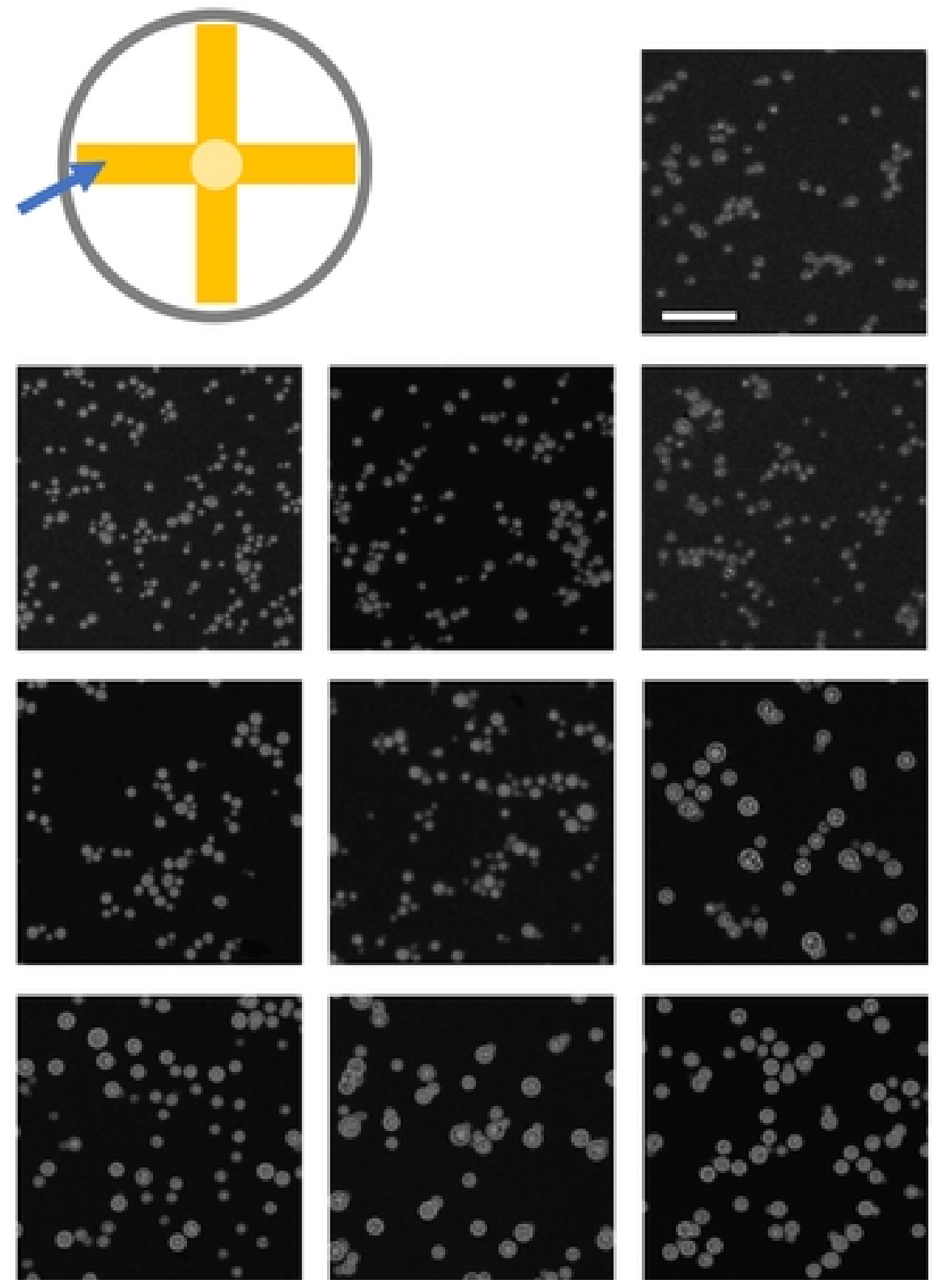

Figure 3 
A
D H99

Ftc555-1

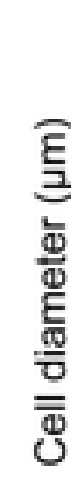

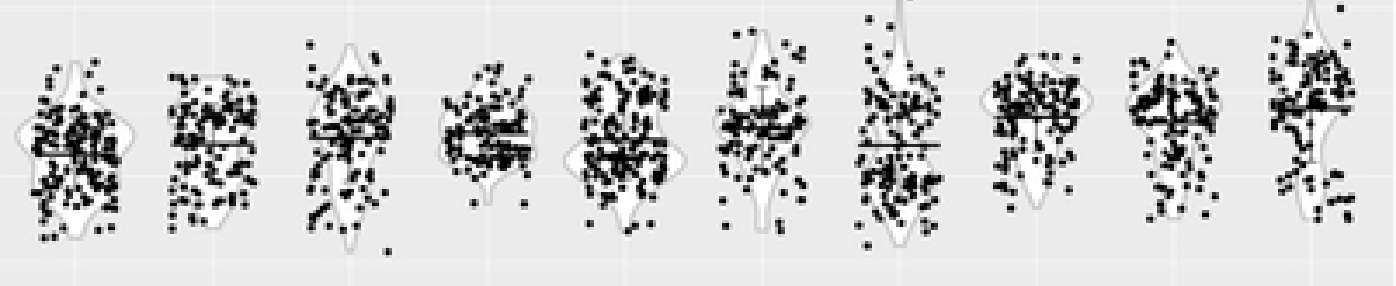
$\begin{array}{llllllllll}\text { A } & \text { C1 } & \text { C2 } & \text { C3 } & \mathrm{H} 1 & \mathrm{H} 2 & \mathrm{H} 14 & \mathrm{H} 13 & \mathrm{H} 16 & \mathrm{H} 17\end{array}$

B

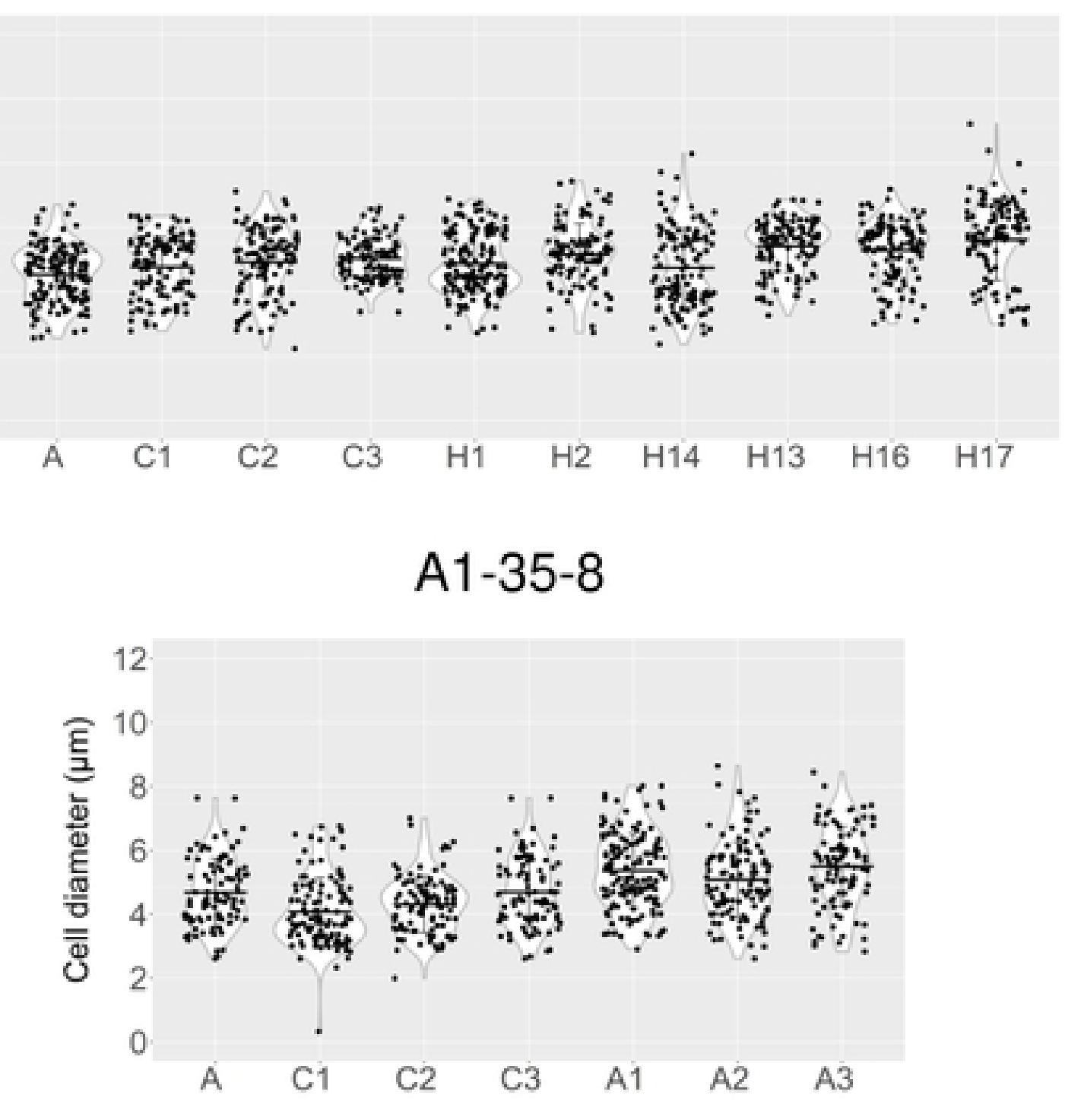

C 


\section{Figure 6}

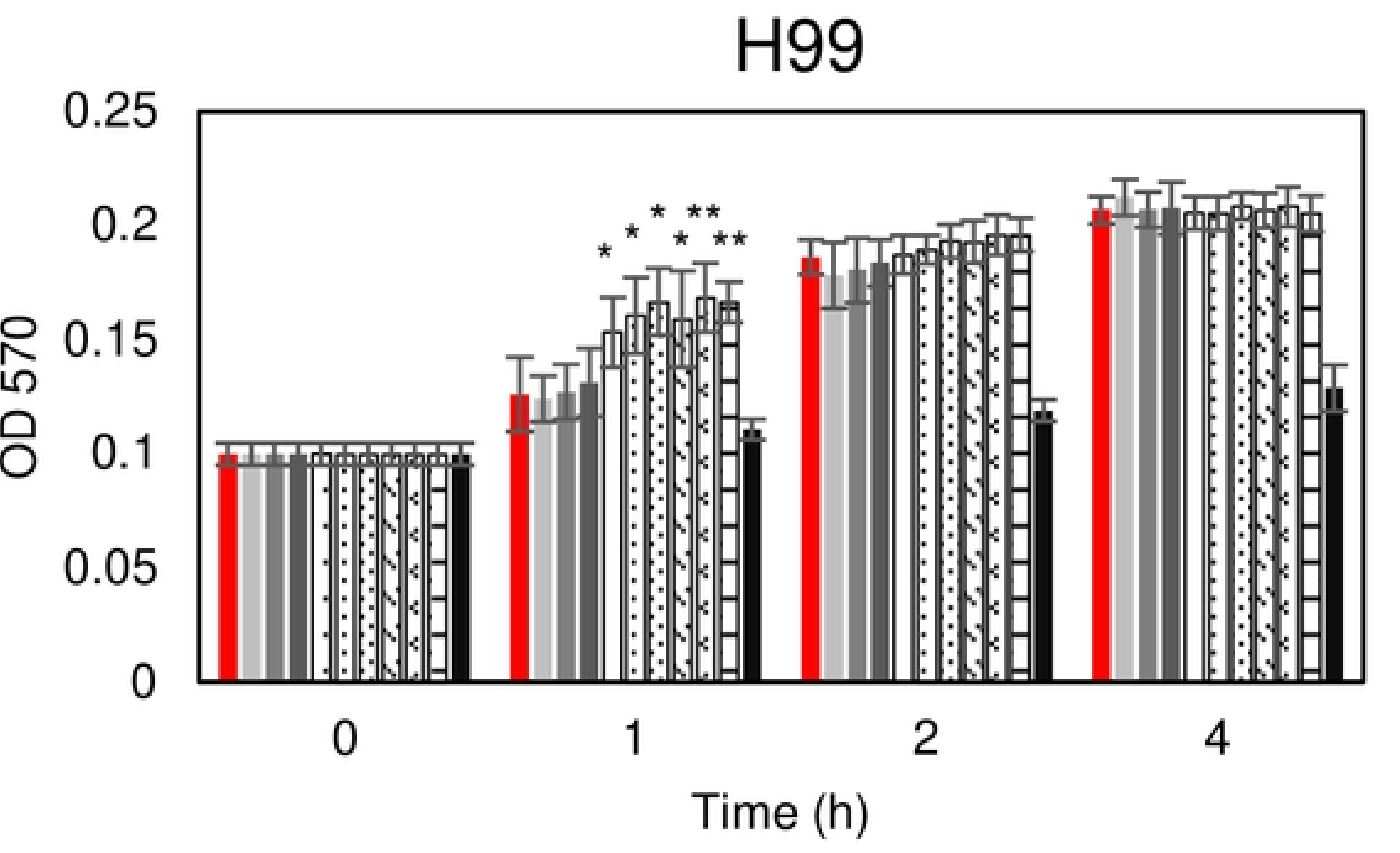

- A

- C1

- $\mathrm{C} 2$

-C3

口 1

口 $\mathrm{H} 2$

口 144

口 13

口 16

다17

- No cell

bioRxiv preprint dhe copyright holder for this preprint

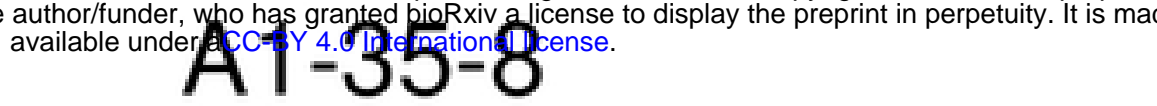

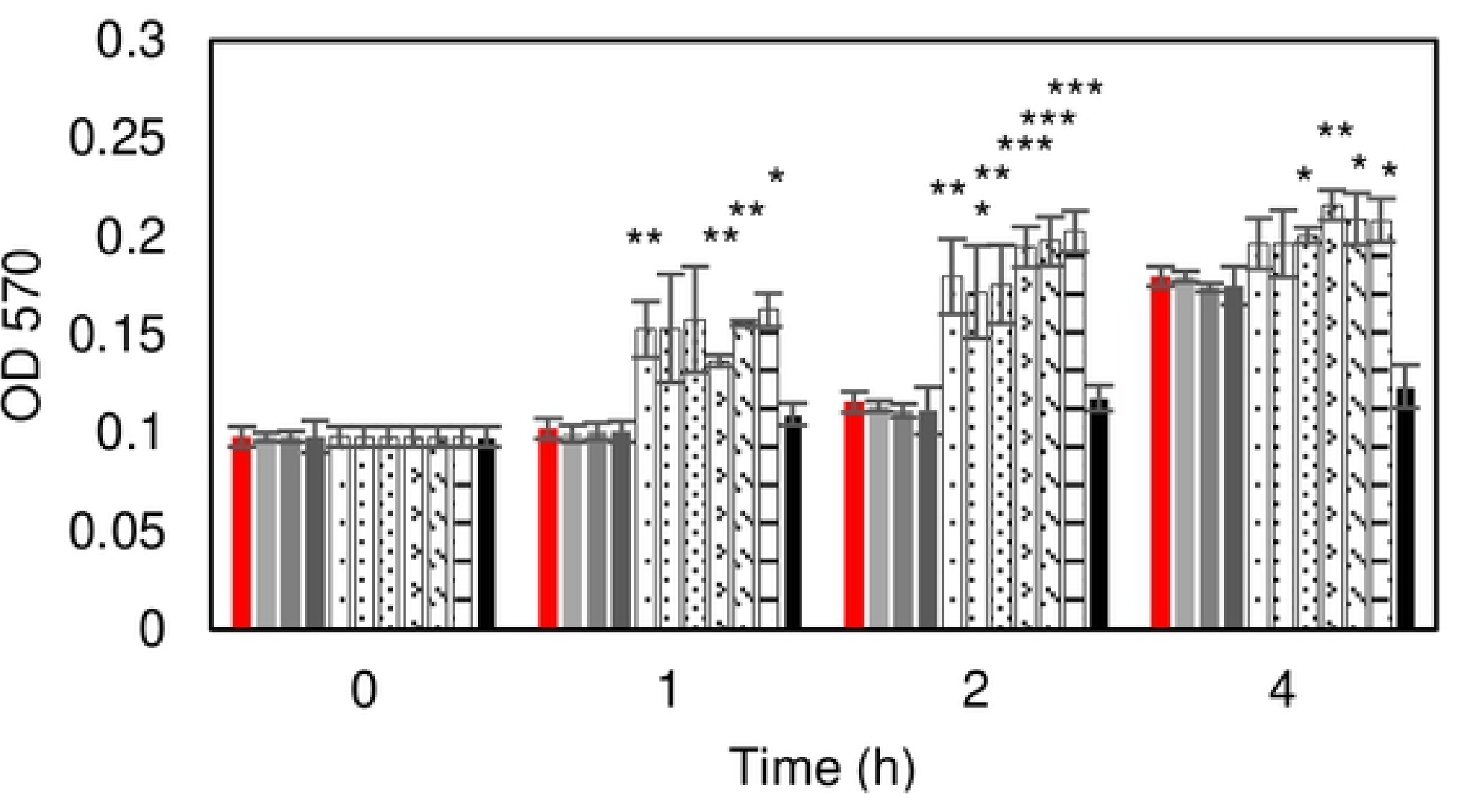

$\because \mathrm{A}$

$\pm \mathrm{C} 1$

$=\mathrm{C} 2$

-C3

$\boxminus \mathrm{A} 1$

$\square \mathrm{A} 2$

$\square \mathrm{A} 3$

$\square \mathrm{A} 4$

$\square \mathrm{A5}$

$\boxminus \mathrm{A} 6$

- No cell

Ftc555-1

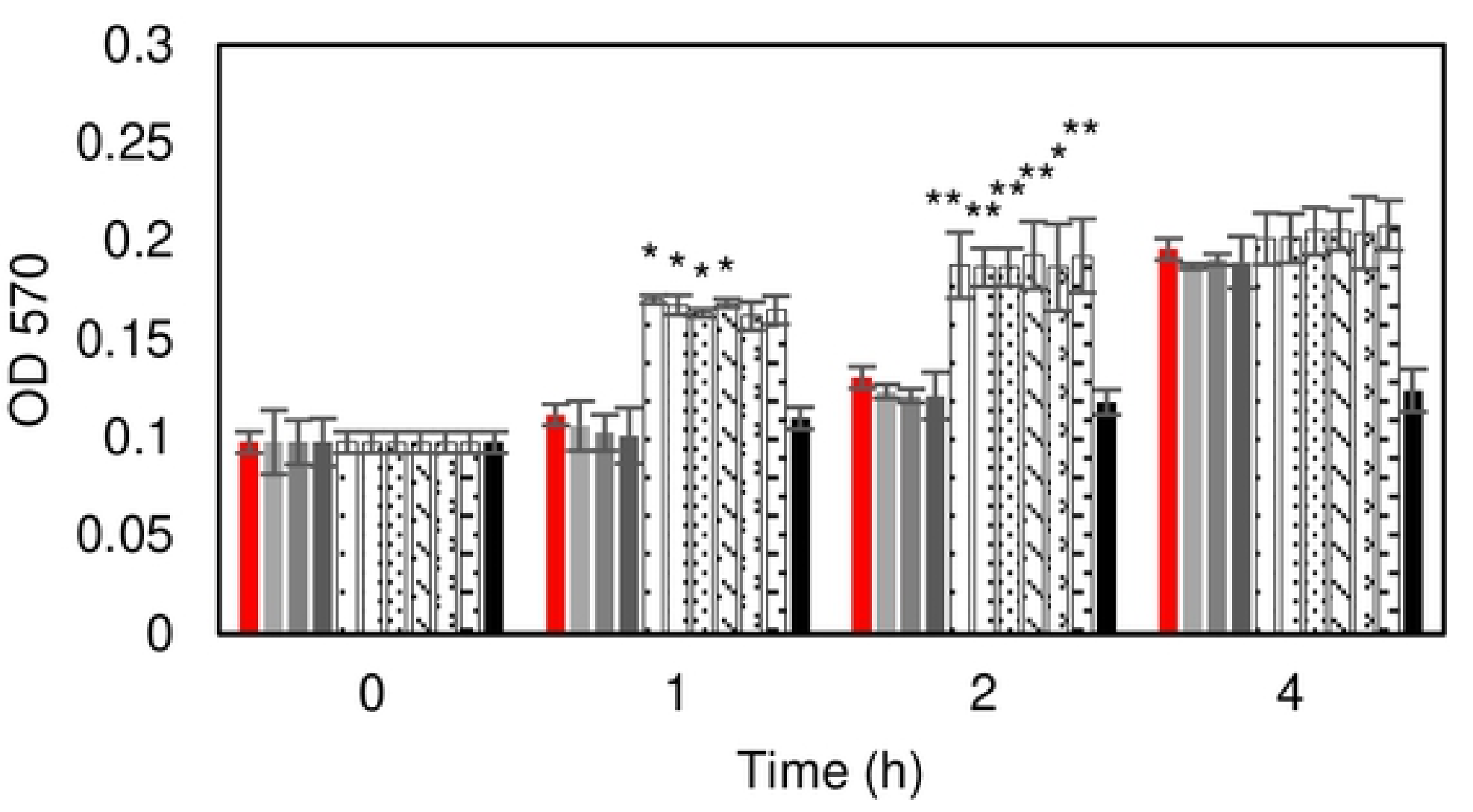

a

= $\mathrm{C} 1$

= $\mathrm{C} 2$

aC3

$\square \mathrm{F} 1$

口F2

बF3

$\square \mathrm{F} 4$

다 5

$\square \mathrm{F} 6$

-No cell

Figure 6 
Figure 8

$30^{\circ} \mathrm{C}+$

$30^{\circ} \mathrm{C}$

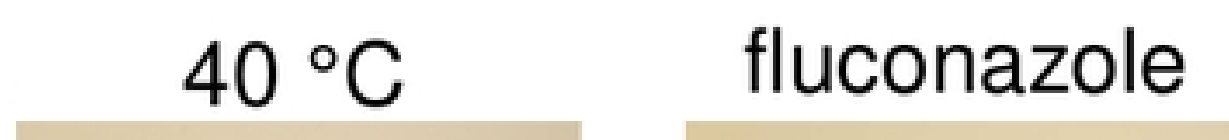

$\mathrm{H} 1$

$\mathrm{H} 2$

$\mathrm{H} 14$

$\mathrm{H} 13$

$\mathrm{H} 16$

$\mathrm{H} 17$

C1

$\mathrm{C} 2$

C3

A

bioRxiv preprint doi: https://doi.org/10.1101/2020.08.07.241190; this version posted August 7, 2020. The copyright holder for this preprint available under aCC-BY 4.0 International license.

A1
A2
A3
A4
A5
A6
C1
C2
C3
A

$\mathrm{F}$

$\mathrm{F} 2$

$\mathrm{F} 3$

$\mathrm{F} 4$

$\mathrm{F} 5$

F6

$\mathrm{C} 1$

$\mathrm{C2}$

C3

A
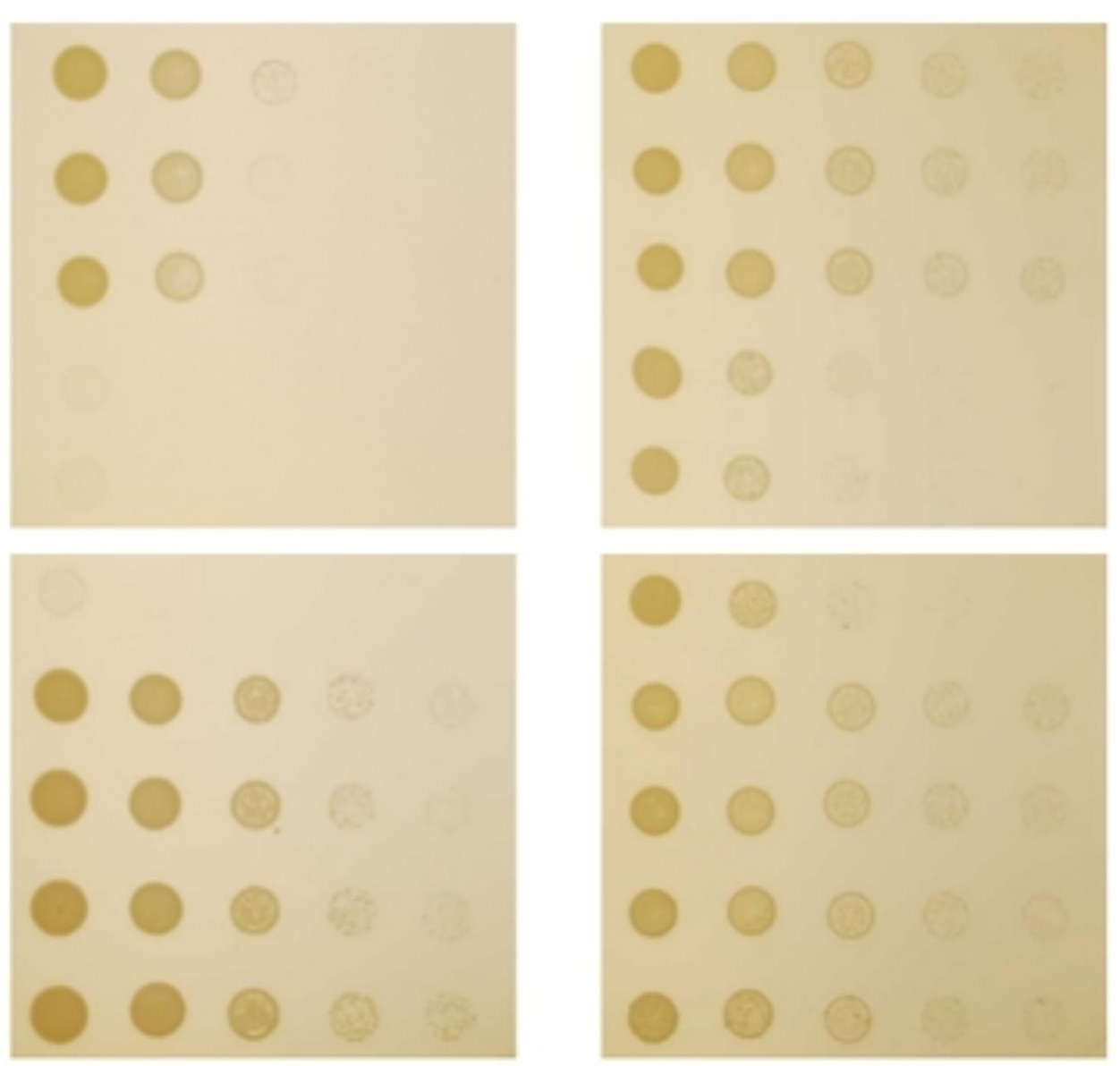

Figure 8 
A

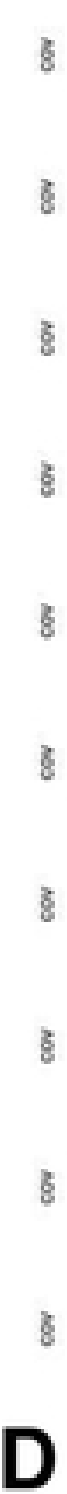

H1

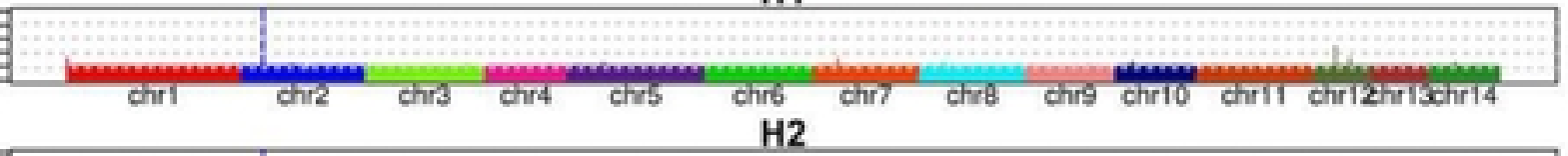

8

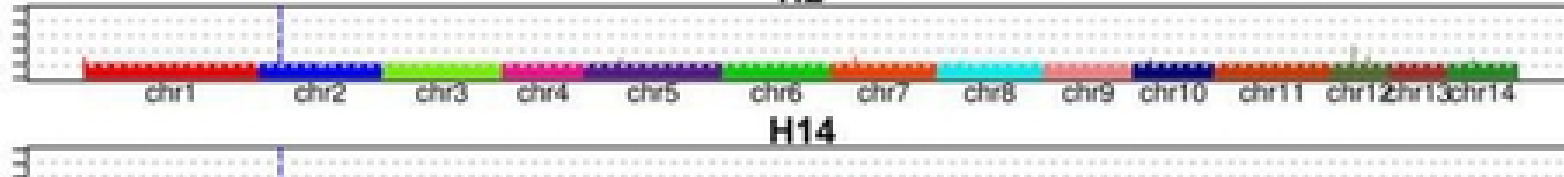

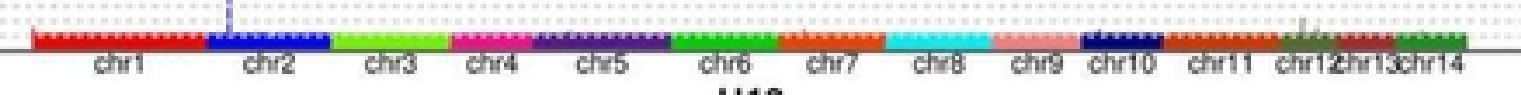
H13

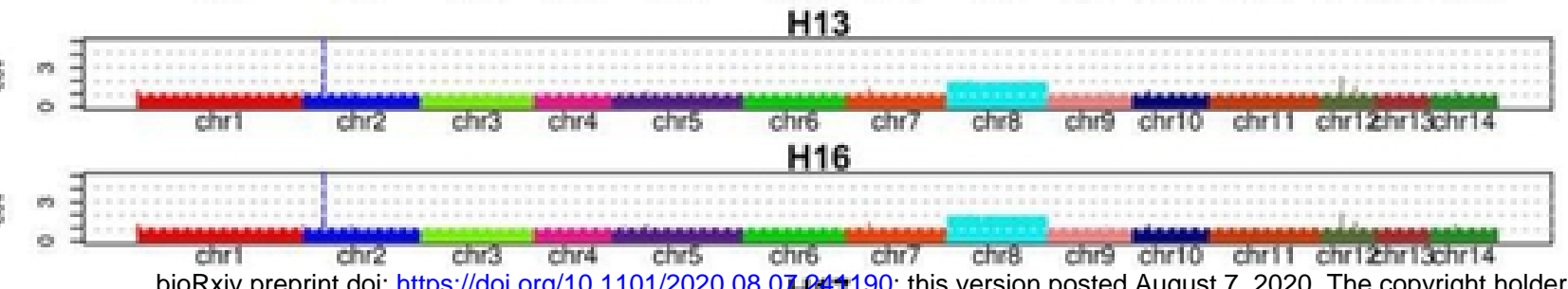

bioRxiv preprint doi: https://doi org/10 1101/2020.08.07 f4t 190; this version posted August 7, 2020. The copyright holder for this eprint

o 3 (which was not certified by peer review) is the authortfunder, who has granted bioRxiv a ticense

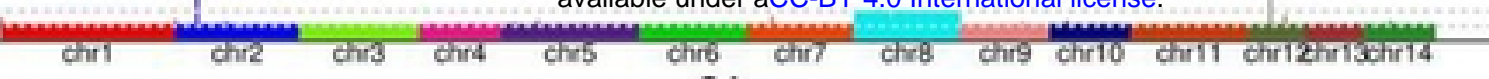
C1

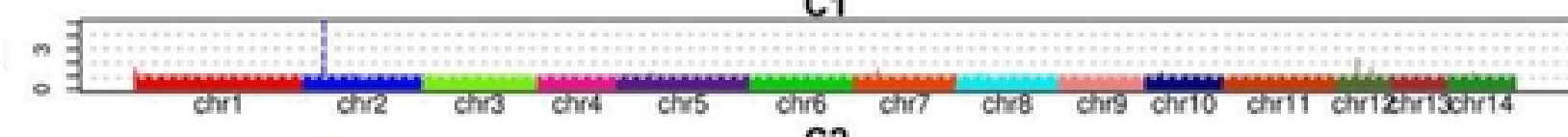
C2 C3

A

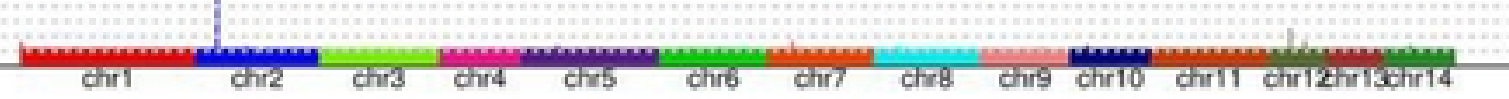

$\mathrm{H} 17^{\mathrm{eu}}$

$\mathrm{H} 17^{\text {aneu }}$
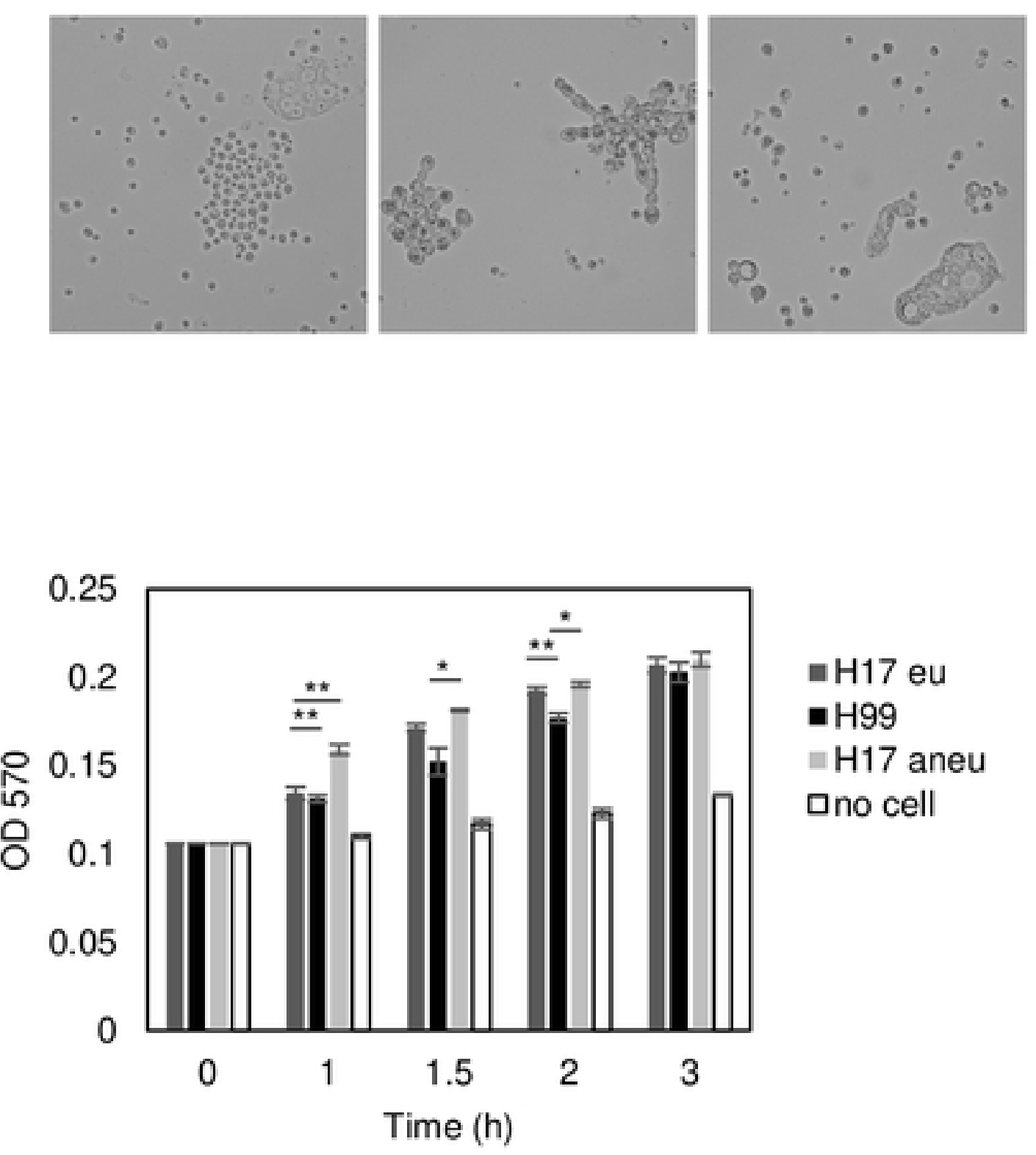

Figure 9
H99

B

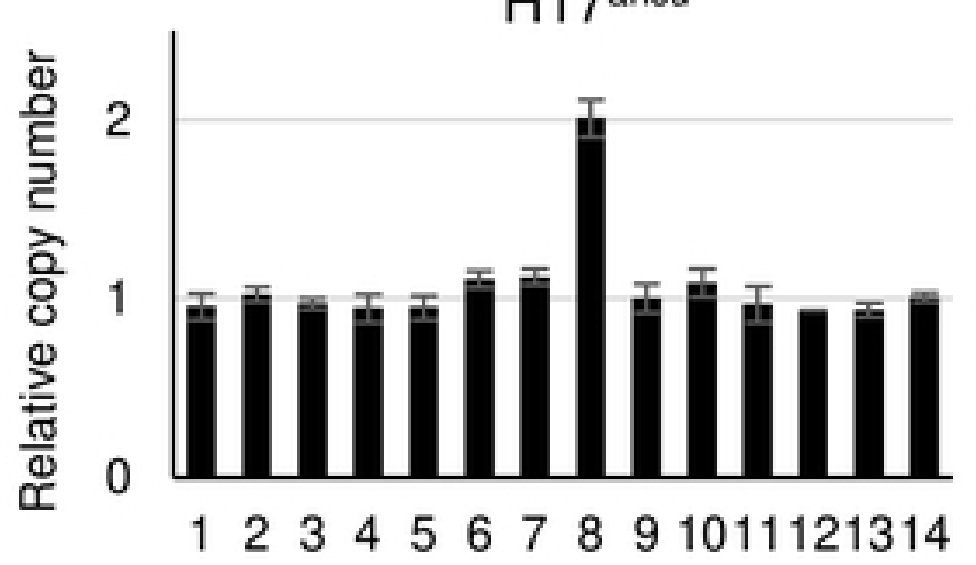
Chromosome

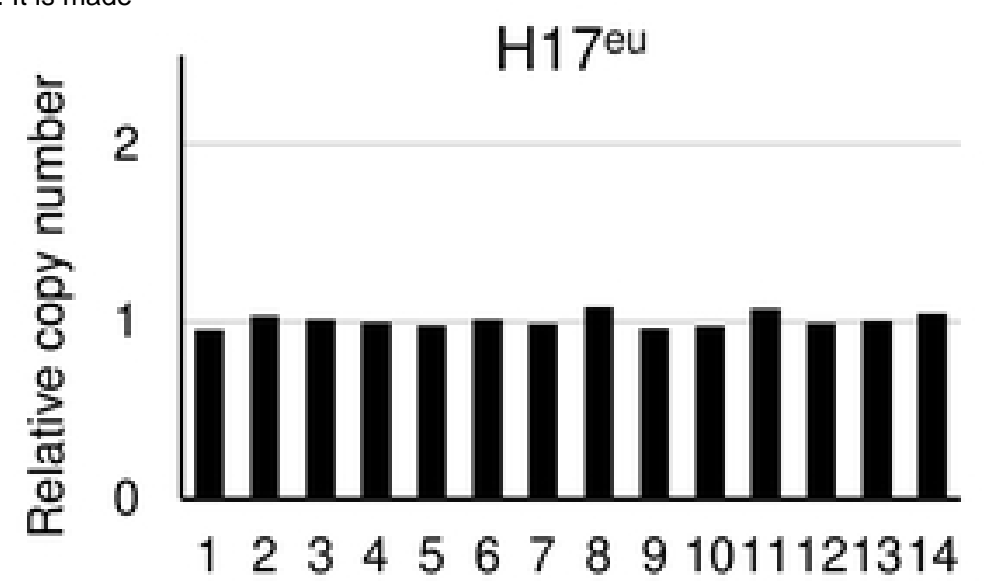

Chromosome

E

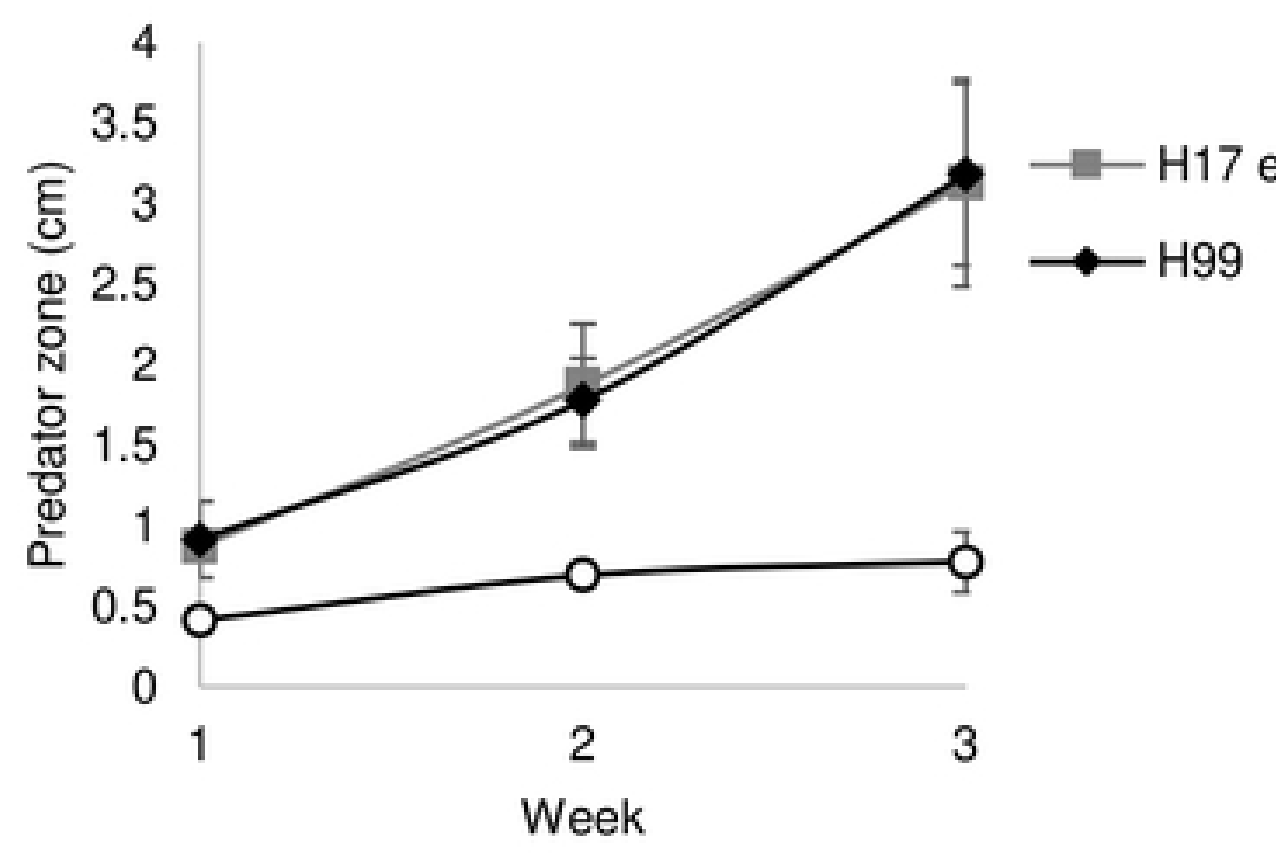

G

- $\mathrm{H} 17 \mathrm{eu}$

- H99

= $\mathrm{H} 17$ aneu

口no cell

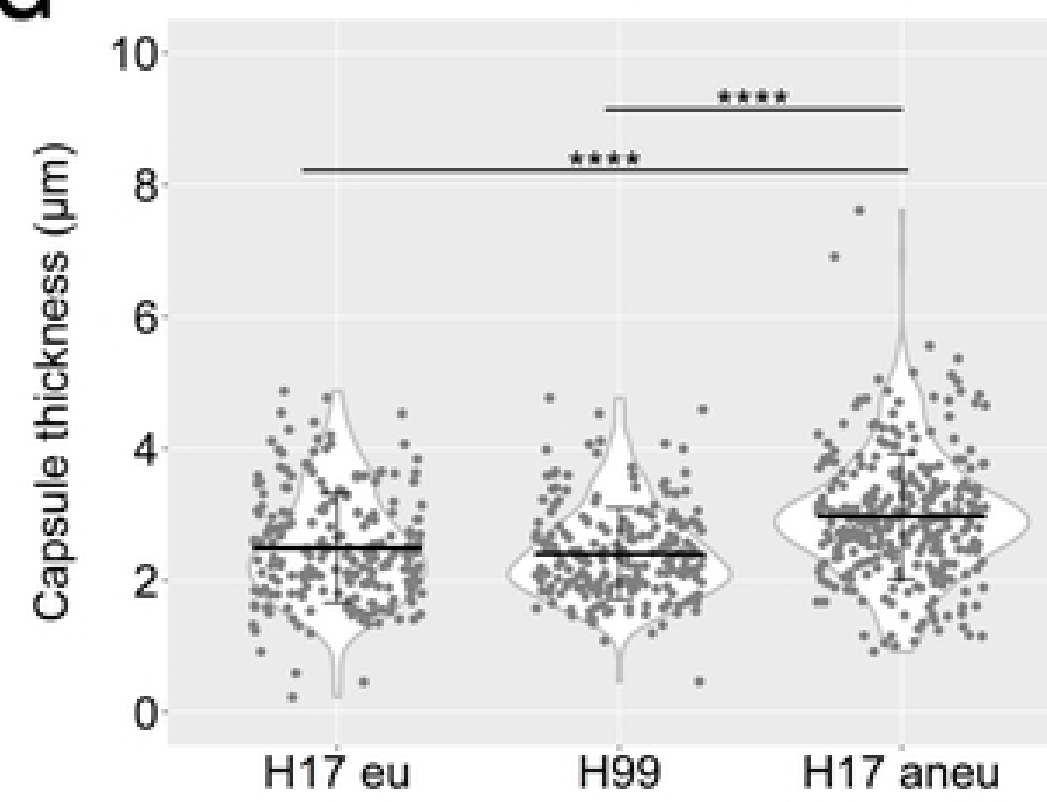


Figure 10
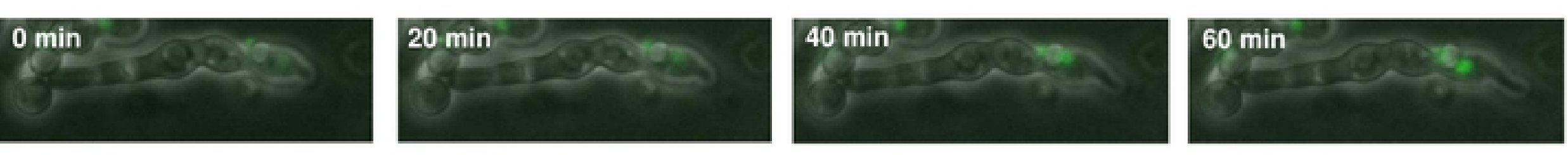

$80 \mathrm{~min}$

$200 \mathrm{~min}$

$220 \mathrm{~min}$

200 min

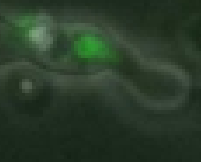

$220 \mathrm{~min}$

$240 \mathrm{~min}$

$260 \mathrm{~min}$

$280 \mathrm{~min}$

\section{$300 \mathrm{~min}$}

$320 \mathrm{~min}$

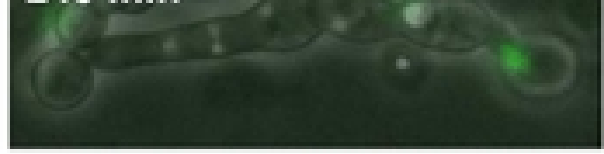

\section{0 min}

$340 \mathrm{~min}$

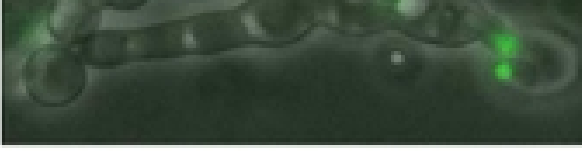

$340 \mathrm{~min}$
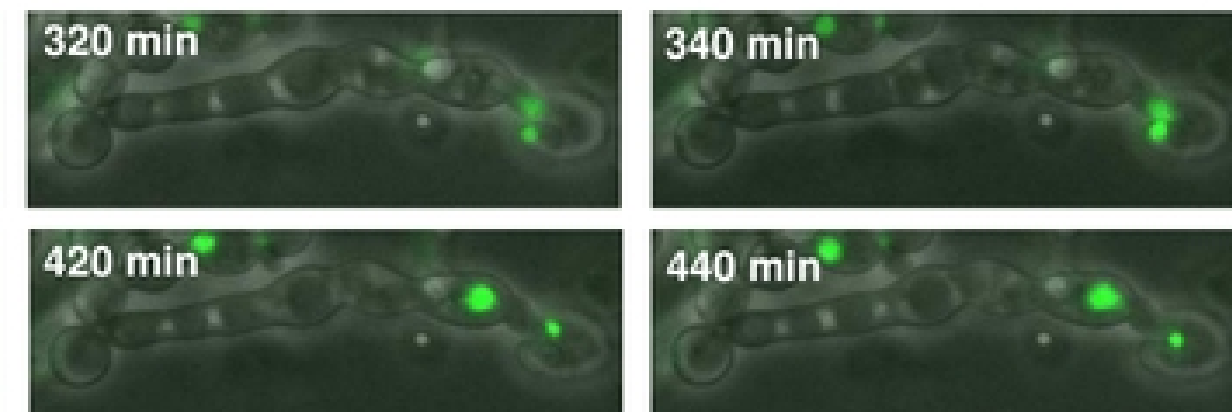

\section{0 min $^{\circ}$}
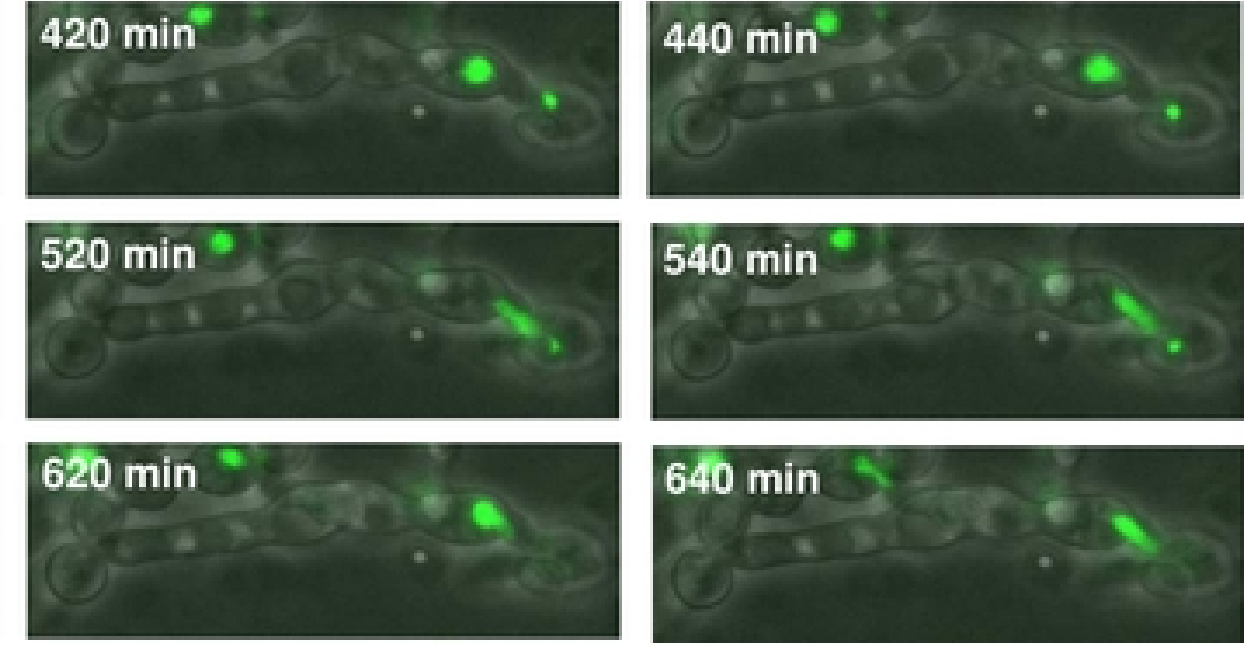

$360 \mathrm{~min}$
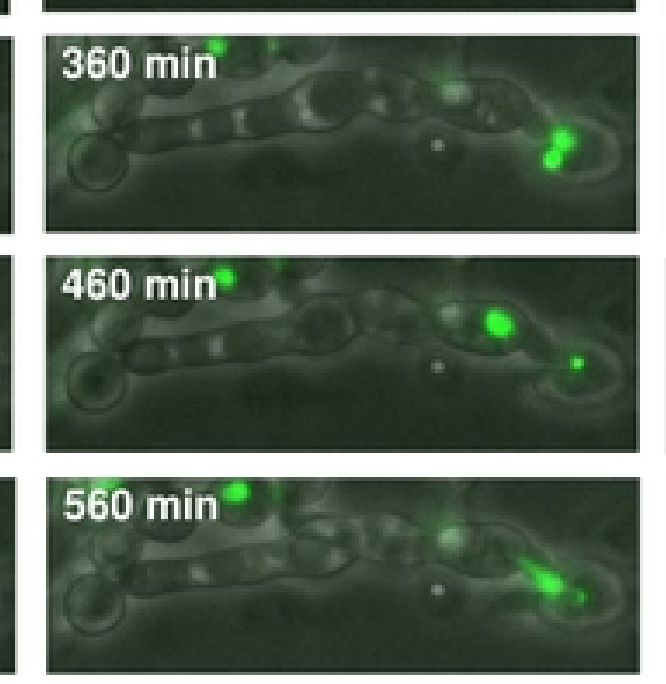

$660 \mathrm{~min}$

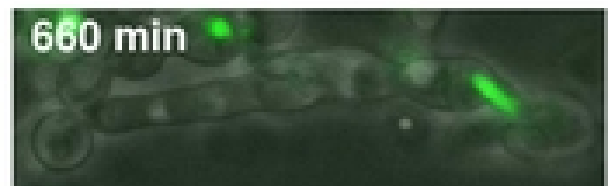

\section{$380 \mathrm{~min}$}

\section{$480 \mathrm{~min}^{\circ}$}

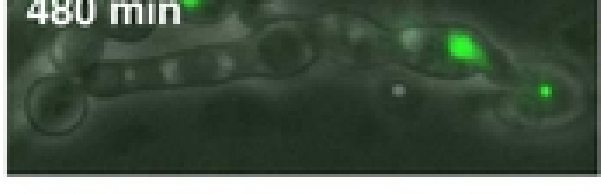

$580 \mathrm{~min}^{2}$

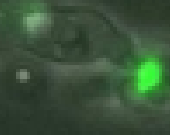

$680 \mathrm{~min}$

\section{Figure 10}


A

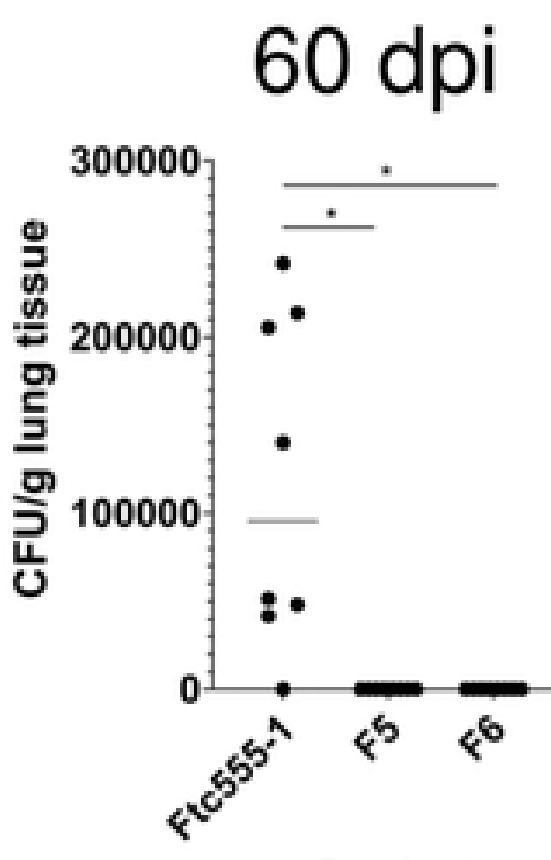

Strains

D

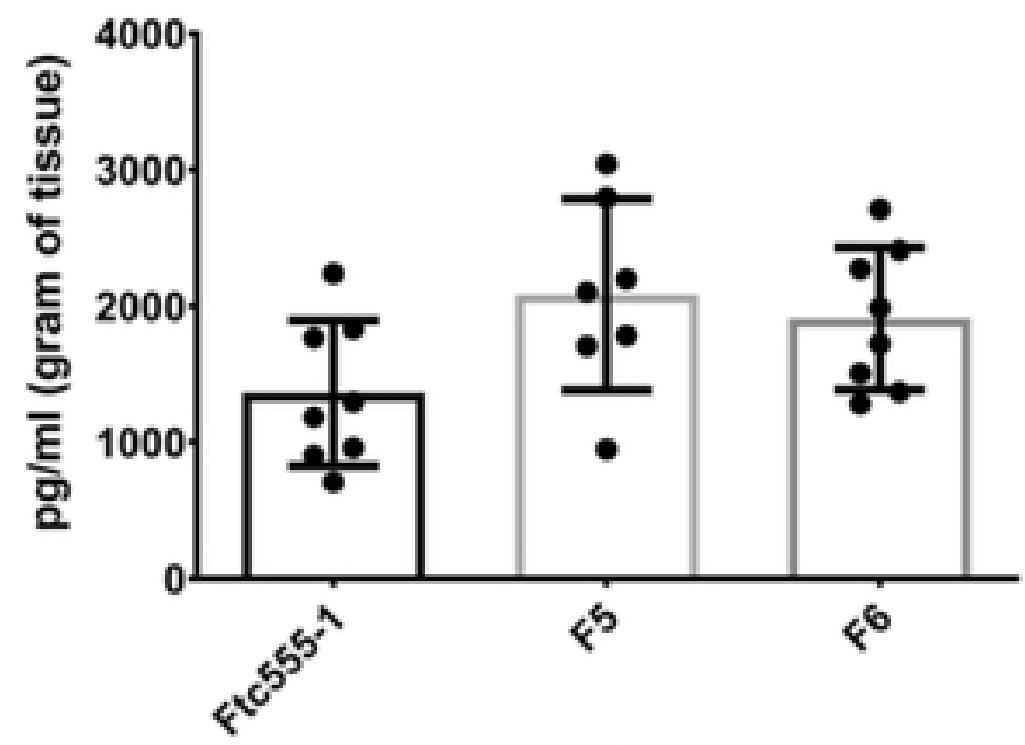

Strains

IFN- $\gamma$

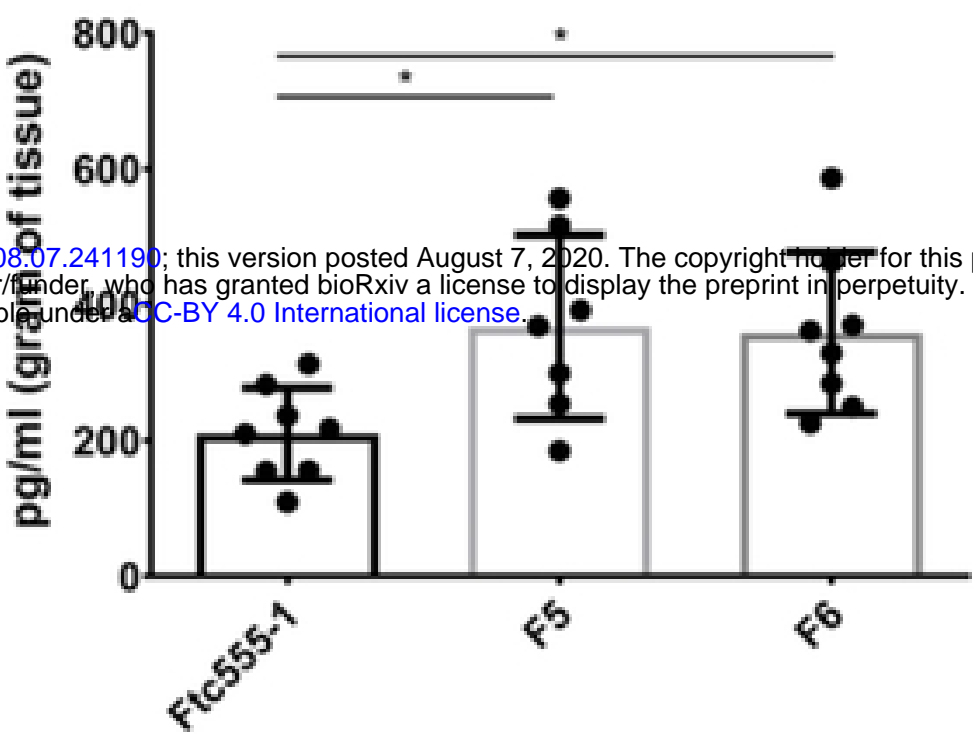

Strains

5 dpi

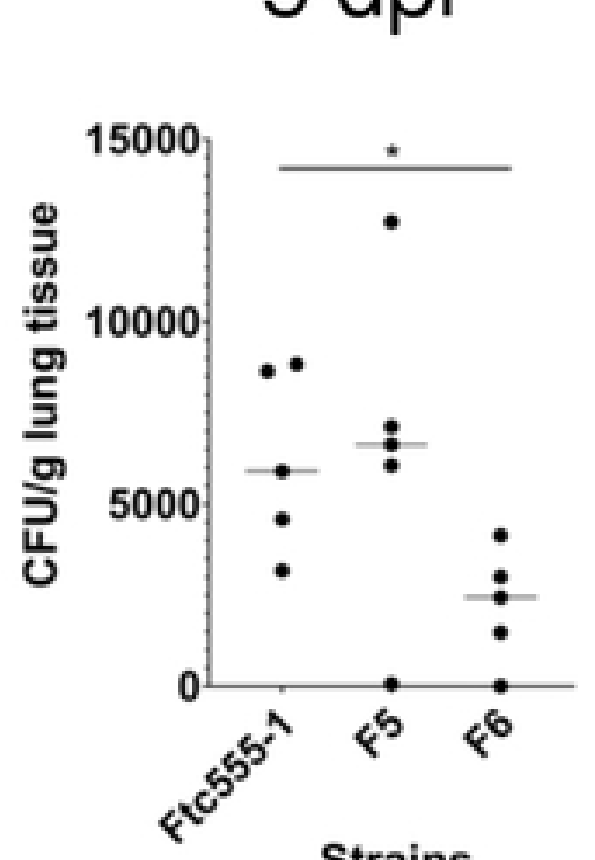

G

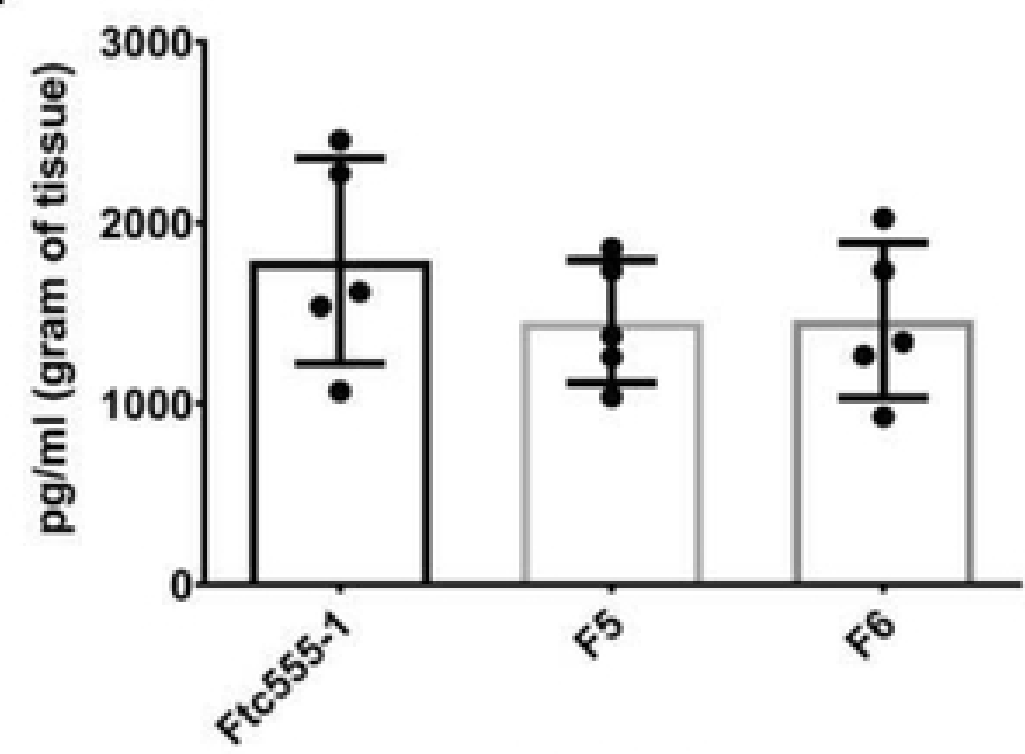

Strains

1

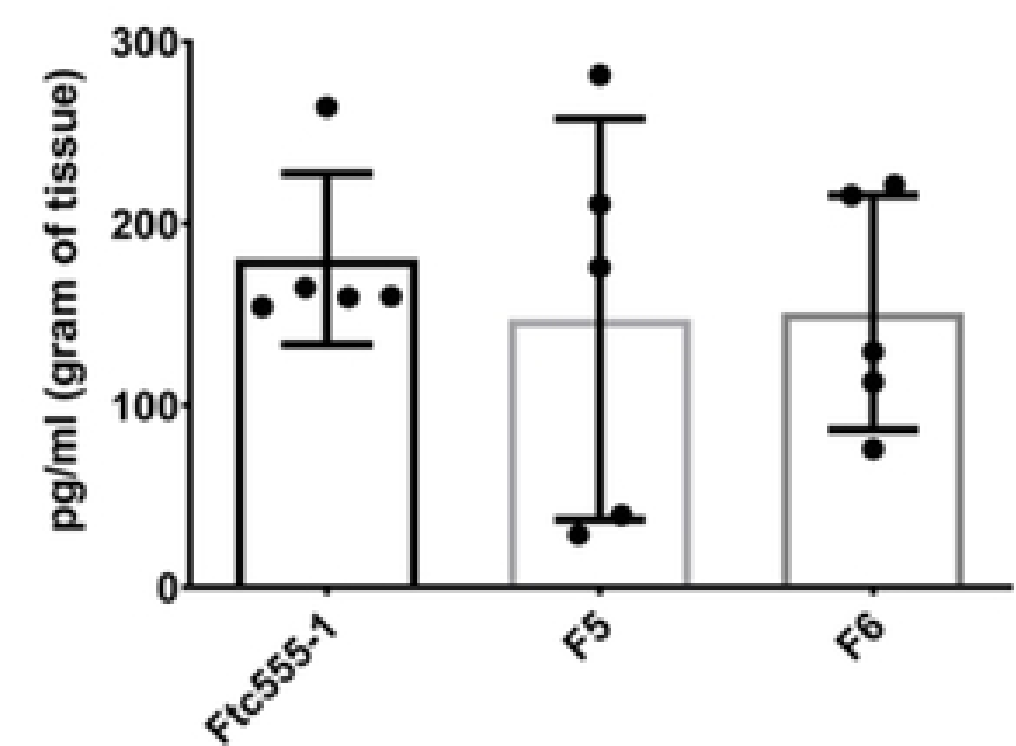

Strains

IL-10

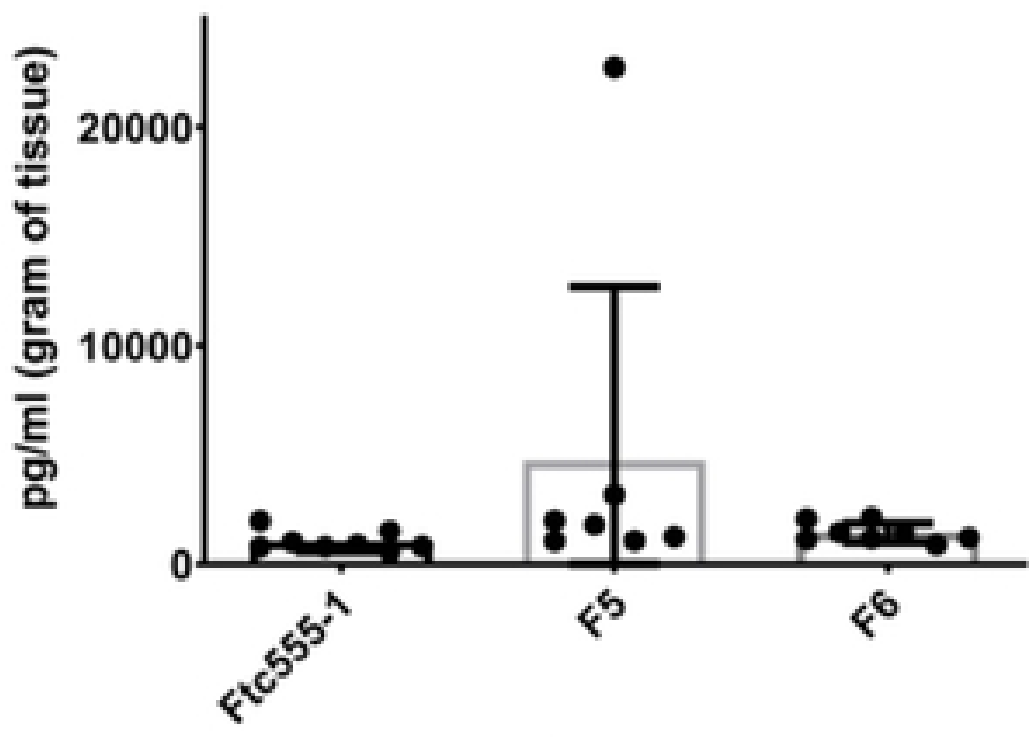

Strains

TNF- $\alpha$

트

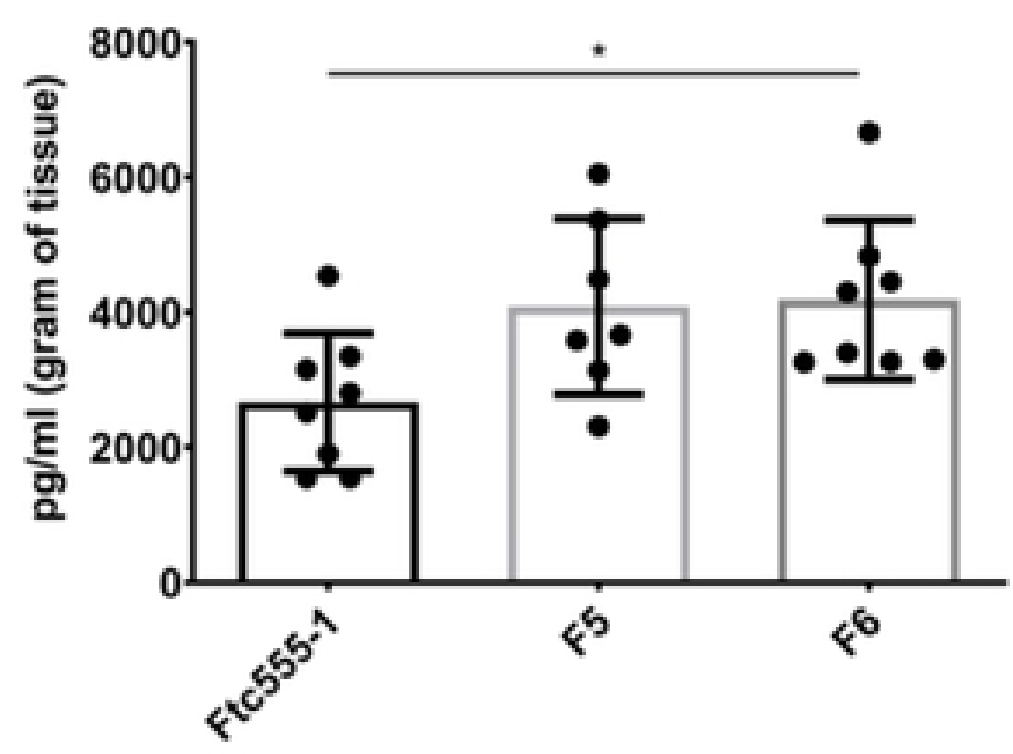

Strains

IL-10

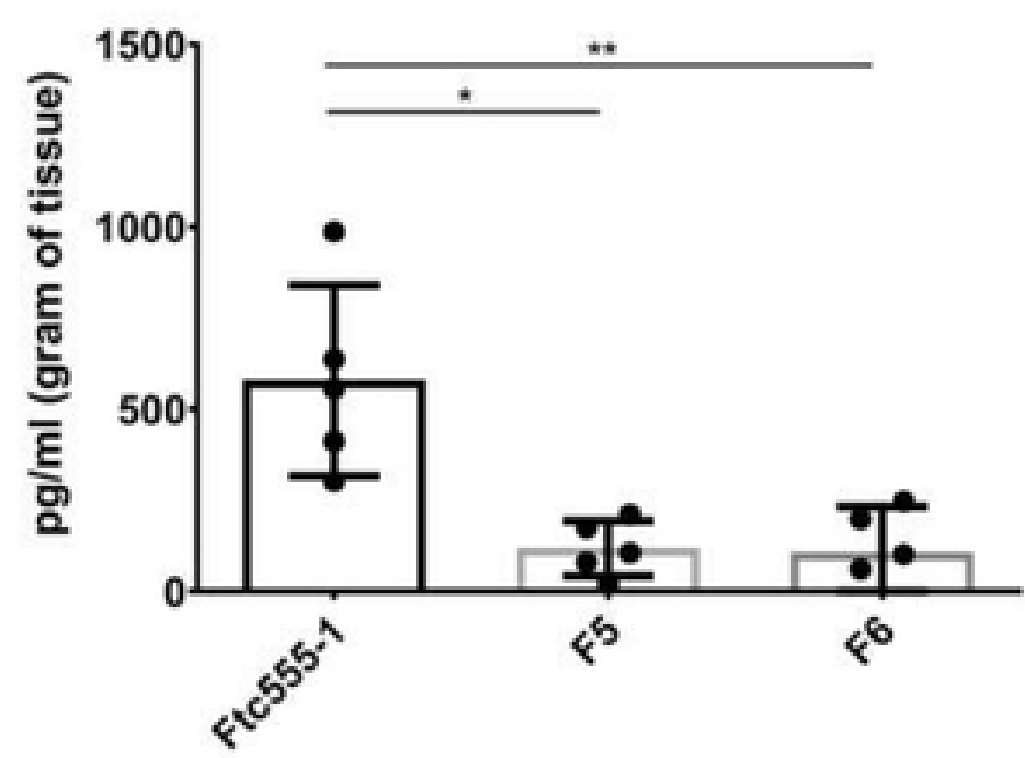

Strains

TNF $-\alpha$

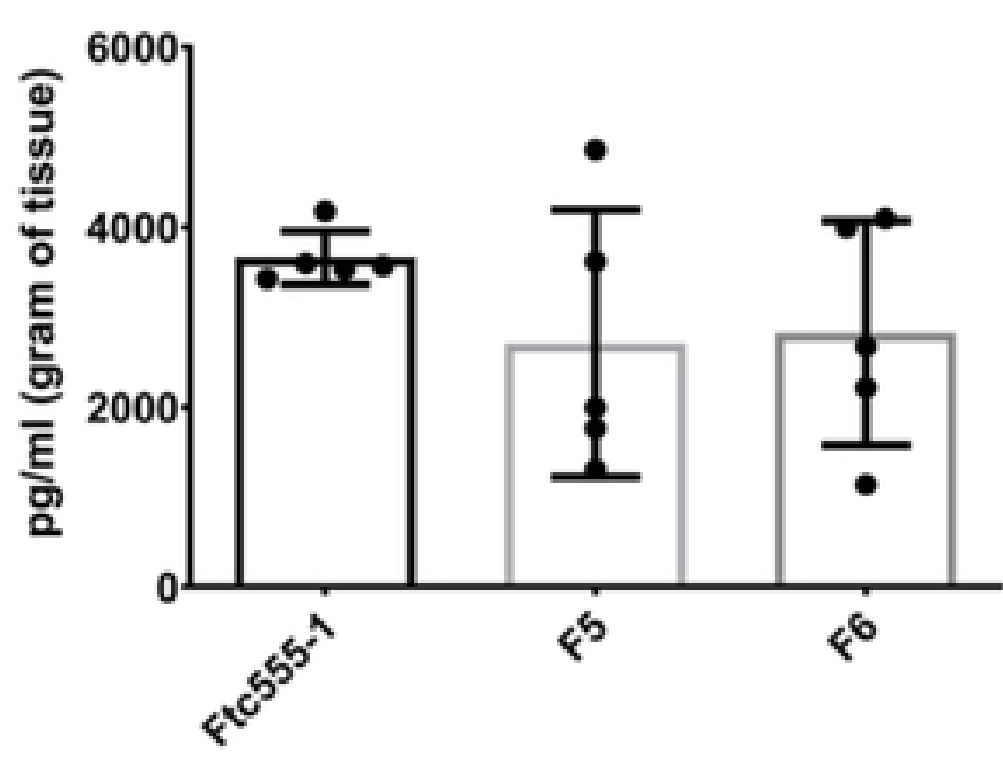

Strains

Figure 12 